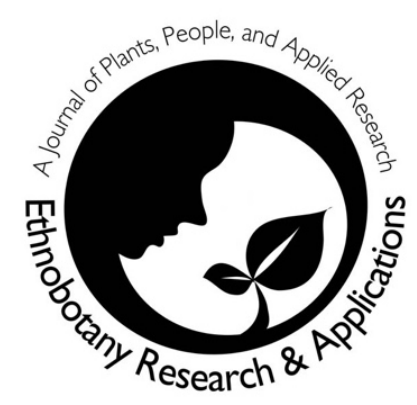

\title{
Ethnopharmacological survey of herbal remedies used in the treatment of hepatitis
}

\author{
Wahid Hussain, Saima Batool, Sabiha Bahar, Bibi \\ Shahida, Bushra Bangash, Amjad Ali, Hidayat Hussain, \\ Iftikhar Ali, Maroof Ali, Rainer W. Bussmann
}

\section{Databases and Inventories}

\begin{abstract}
Background: Hepatitis is a serious illness and can be fatal, which has caused a dangerous ratio round the globe especially in the developed and underdeveloping countries. About 1.4 million deaths are reported each year from the different types of hepatitis especially hepatitis B virus (HBV) and hepatitis $\mathrm{C}$ virus (HCV) which cause $90 \%$ mortality and the other $10 \%$ deaths are caused due to all other hepatitis types.
\end{abstract}

Methods: By using different online database such as Google scholar, Science Direct Navigator, ISI Web of Knowledge, Elsevier, springer link, Test databases, research gate and PubMed, date were retrieved by giving different keywords. Various keywords were used on plants used traditionally to treat hepatitis.

Results: A total of 128 medicinal plant species were identified, belonging to 108 genera and 61 botanical families by reviewing 220 research articles. From the study, it showed that the medicinal plants are used from the very ancient time to cure hepatitis worldwide. These medicinal plants are used in different parts of the world as a traditional herbal medicine for the control of hepatitis.

Conclusion: Our literature review will help the scientific communities to identify anti-hepatitis plant in order to isolate novel anti-hepatitis natural products.

Keywords: Hepatitis, medicinal plants, treatment, ethnopharmacological, survey

\author{
Correspondence \\ Wahid Hussain $^{1 *}$, Saima Batool ${ }^{1}$, Sabiha Bahar ${ }^{1}$, \\ Bibi Shahida1, Bushra Bangash ${ }^{1}$, Amjad $\mathrm{Ali}^{2}$, \\ Hidayat Hussain ${ }^{3}$, Iftikhar $\mathrm{Ali}^{4}$, Maroof $\mathrm{Ali}^{5}$, Rainer \\ W. Bussmann ${ }^{6}$ \\ ${ }^{1}$ Department of Botany, GPGC Parachinar Kurram \\ Pakistan \\ 2Department of Sustainable Crop Production, \\ Università Cattolica del Sacro Cuore, Via Emilia \\ Parmense, 8429122 Piacenza Italy \\ ${ }^{3}$ Department of Bioorganic Chemistry, Leibniz \\ Institute of Plant Biochemistry Weinberg 3, D-06120 \\ Halle (Saale), Germany \\ ${ }^{4}$ Shandong Analysis and Test Center, Shandong \\ Provience,(250014), China \\ ${ }^{5}$ College of Life Science, Anhui Normal University, \\ Wuhu 241000, China \\ ${ }^{6}$ Institute of Botany, Department of Ethnobotany, Ilia \\ State University, Georgia \\ Corresponding Author: \\ wahidhussainwahid@gmail.com \\ Ethnobotany Research \& Applications \\ 20:24 (2020)
}

خلاصس:

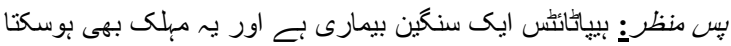

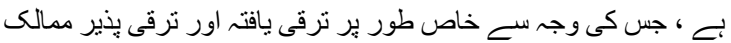

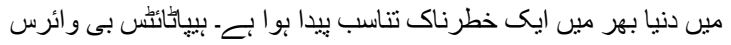

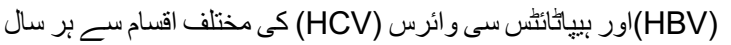

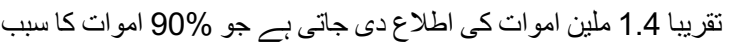

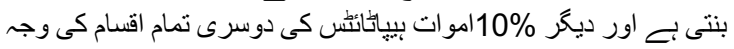

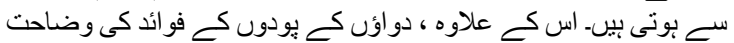

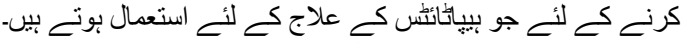

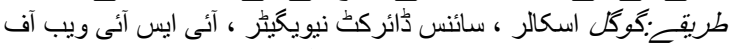

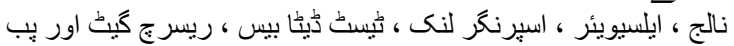




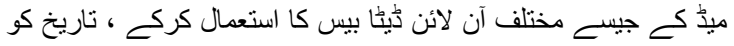

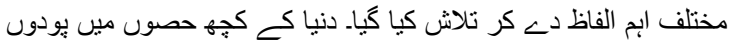

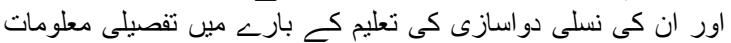

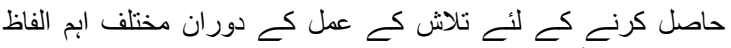
استعمال كيه كئه

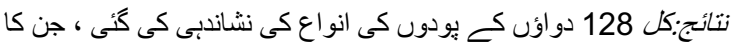

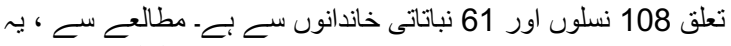

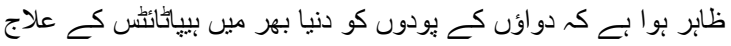

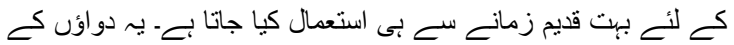

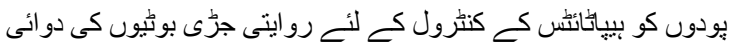

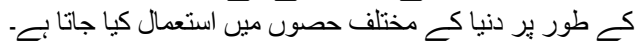

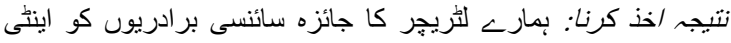

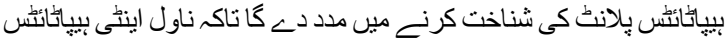

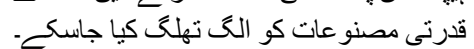

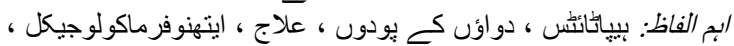

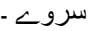

\section{Background}

The liver is an organ and paramount importance. It aids in the metabolism of ingested substances like food, alcohol, dietary substances and most medications (Govind and Pandey 2011, Thomas et al. 2011). There are many causes of liver disorders, but the major causes are various toxic chemicals, some antibiotics, alcohol, and food. In addition, liver disorders are also caused due to the infections of various kinds of parasites, viruses, fungi or bacteria. Different liver disorders like hepatitis, jaundice, inflammation of liver, cirrhosis (a digestive disorder that is the result of liver fibrosis), and liver cancer also be caused by autoimmune distractions (Govind \& Pandey 2011). The word hepatitis originates from two Greek words, hepta that refers to liver and it is meaning inflammation. Hepatitis is present in many forms such as including virus hepatitis, autoimmune hepatitis, fatty liver hepatitis, alcoholic hepatitis and toxin induced hepatitis these all types of hepatitis is identified as a hepatitis virus that attack and harm liver cells (Khan et al. 2010).

The Hepatitis B virus was first studied in 1963 (Cusheri 2002) and it is estimated that more than two billion people have ate some point been infected with HBV on global level (World Health Organization 2000. About, 520000 patients annually from hepatitis type B and C (International consensus conference 2002). Hepatitis C was first cloned (Choo et al. 1989, Alamo et al. 2013). About 170 million people are affected by hepatitis $\mathrm{C}$ virus, according to World Health Organization (WHO), which causes liver cirrhosis or hepatocellular carcinoma (Chen \& Morgan 2006). The percentage of prevailing HBV and HCV infections among the people of Pakistan is $35-38 \%$ and $4-14 \%$ respectively (Hakim et al. 2008). Pakistan is confronting a huge burden of these diseases. Almost 30 genotypes of HCV have been describing until now (Kato 2000). HCV causes a chronic liver disease responsible 8000-10000 deaths in Pakistan per year (Moyer et al. 1999).

All hepatitis viruses have different ways of transmission. For instance, the hepatitis $A$ virus is easily transmitted under unhygienic condition and in poor sanitation. Hepatitis B virus (HBV) is transmitted via blood and also in some case it transmitted from the blood-derived products (Seeger et al. 2020).

There is a complex biological and cultural relationship between plants and human beings (Balick 1996) and herbal medicines have a great role in the discovery of different drugs, and they are used for the cure of different disorders all over the world. Usually these sources are available in abundance and traditional medicines benefits 80 percent of population on earth (Gewali \& Awake 2008, Wangchuk et al. 2011). In modern pharmacopoeia, almost 25 percent of all drugs were derived from plants (Phytomedicines) and other are synthetic analogous of natural products (Pseudo natural products) built on prototype compounds derived from plants (Bandaranayake 2006). Several medicinal plants and their formulations have been used around the world for the treatment of liver diseases and this has encouraged the scientific community to research the effect of these medicinal plants which can treat $\mathrm{HCV}$ in a comprehensive manner (Ashfaq \& Idress 2014). Many drugs have been reported against HCV until date and many of them are found successful in clinical trials, but viral resistance and the negative effects of these drugs have made their therapeutical application against $\mathrm{HCV}$ limited (Kitazato et al. 2007). The effect of Acacia nilotica (AN) in inhibition of $\mathrm{HCV}$ and of other plants e.g. against herpes simplex virus (HSV), poliovirus type 1, coxsackievirus B5 and Echovirus have been confirmed (Konowalchuk and Spears 1976). Our study aims to elicit plant species used against liver disorders especially hepatitis.

\section{Materials and Methods}

By using different online database such as Google scholar, Science Direct Navigator, ISI Web of Knowledge, Elsevier, springer link, Test databases, research gate and PubMed, date were retrieved by using different keywords in order to derive detailed information of plants and their ethnopharmacological studies of various regions of the world (Adnan at el. 2014). With the help of taxonomic literature, botanical names of plants and their families were verified (Ullah et al. 2018).

\section{Data analysis}

Data were compared using Venn diagrams and calculating the Jaccard (similarity) Index (JI) of each 
couple of datasets (Jaccard 1902, Kayani et al. 2015).

To determine the similarity between two sets the following formula was used:

Jaccard Index $=$ (the number in both sets) $/$ (the number in either set) * 100

The formula in notation is as follows:

$$
J(X, Y)=|X \cap Y| / \mid X \cup Y{ }^{*} 100
$$

Moreover, the recorded data were also compared with the wild food ethnobotanical literature of Pakistan in order to assess their possible novelty (Abbas et al. 2020, Ahmad and Pieroni 2016, Ahmad et al. 2019).

\section{Family Importance Value}

FIV represents the comparative position of families. It provides the regional prominence of the species. The intention was to compel the percentage that came from informants who mentioned the family as follows;

$$
F I V=\frac{F C}{N} \times 100
$$

Where FC is the sum of informers revealing the family, while $\mathrm{N}$ is the sum of informants who participated in the research.

\section{Results and Discussion}

Diversity of the medicinal flora

During the current review, a total of 128 taxa belonged to 108 genera, from 66 plant families were documented to be consumed as medication for hepatitis diseases all over the world (Fig. 1).

There was only one family of fungi, pteridophytes, and gymnosperms with one species each, and 125 species belonging to 63 angiosperm families. Plant species were laterally registered with the corresponding family, botanical names, local names, part used, preparation of different remedies, route of administration, and medicinal uses as mentioned in Table 1.

\section{Plant Parts Used}

Based on a total of 191 citation reports, the part of the plants most frequently used for curing hepatitis were leaves $(27 \%)$, followed by aerial parts $(18 \%)$, fruit $(16 \%)$, fruit $(15.7 \%)$, flower $(11 \%)$, root $(10 \%)$, seed $(7 \%)$, stem $(5 \%)$,bulb, bark, rhizome each $(1 \%)$, fruiting body and flower top $(1 \%)$ as shown in Figure 2. The least reported plant part used bulb, bark, rhizome each (1\%), fruiting body and flower top each with 1 percent.

\section{Family Importance Value}

The importance of a plant family increases with the increase in the frequency of citations of its all species. Table 1 represents 66 plant families with maximum FIV, amongst which Asteraceae was the dominant family (13.8\%), followed by Fabaceae $(7.8 \%)$, Euphorbiaceae $(2.7) \%$, Cucurbitaceae $(2.5 \%)$ and Gentianaceae (2.0\%). Acanthaceae, Amaryllidaceae, Apiaceae, Brassicaeae and Poaceae had (1.5\%) family importance values. Rest of the families had less than (1.5\%) family importance values as shown in Figure 3.
61

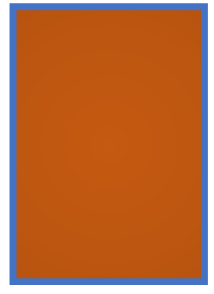

Family

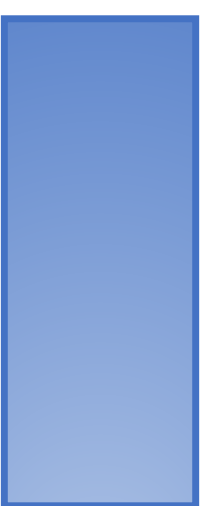

Genera

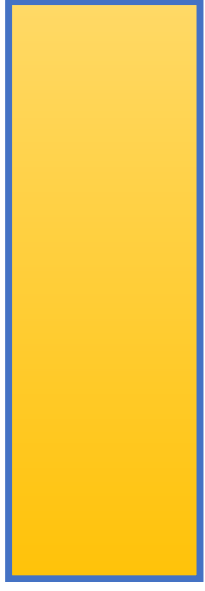

Species

Figure 1. Diversity of medicinal flora used for the treatment of hepatitis 
Table 1. List of the plants used for the treatment of various types of hepatitis all over the world

\begin{tabular}{|c|c|c|c|c|c|}
\hline Family & Plant Name & Part used & Chemical constituents & Uses & Citation \\
\hline \multirow[t]{3}{*}{ Acanthaceae } & Acanthus ilicifolius L. & Leaves & $\begin{array}{l}\text { Aceteoside, isoaceteoside, } \\
\text { acanthaminoside }\end{array}$ & $\begin{array}{l}\text { Used for hepatitis, and also for anti-ulcer } \\
\text { activities }\end{array}$ & $\begin{array}{l}\text { Samani \& Kopaei, } \\
2018, \text { Kumar \& Choyal, } \\
2012\end{array}$ \\
\hline & Justicia adhatoda L. & Roots & $\begin{array}{l}\text { Alkaloids, polyphenolics, } \\
\text { glycosides. }\end{array}$ & $\begin{array}{l}\text { Decotion of its roots is taken by the } \\
\text { patients for one month in the treatment of } \\
\text { jaundice. }\end{array}$ & $\begin{array}{l}\text { Kumar \& Choyal, 2012, } \\
\text { Medeiros et al., } 2012\end{array}$ \\
\hline & $\begin{array}{l}\text { Justicia schimperiana } \\
\text { (Hochst. ex A. Nees) T. } \\
\text { Anderson }\end{array}$ & $\begin{array}{l}\text { Leaves, } \\
\text { Stem }\end{array}$ & $\begin{array}{l}\text { Alkaloids, lignans, flavonoids, and } \\
\text { terpenoids }\end{array}$ & $\begin{array}{l}\text { Chopped and used to take a smoke bath; } \\
\text { boiled and used as a body wash; leaves } \\
\text { and stems are crushed and pounded and } \\
\text { then taken orally }\end{array}$ & $\begin{array}{l}\text { Kpodar et al. 2015, } \\
\text { Corrêa \& Alcântara, } \\
2012\end{array}$ \\
\hline Adiantaceae & $\begin{array}{l}\text { Adiantum capillus-veneris } \\
\text { L. }\end{array}$ & Leaves & $\begin{array}{l}\text { Flavonoids, terpenoids,tannins, } \\
\text { muciliage, volatile oils, gallic acid }\end{array}$ & $\begin{array}{l}\text { Used for hepatitis and also for } \\
\text { inflammatory diseases }\end{array}$ & $\begin{array}{l}\text { Jan et al. 2017, } \\
\text { Prajapati et al. } 2006 \\
\end{array}$ \\
\hline Aizoaceae & $\begin{array}{l}\text { Trianthema } \\
\text { portulacastrum } \mathrm{L} .\end{array}$ & Aerial parts & $\begin{array}{l}\text { Trianthenol, 3-acetylaleuritolic acid, } \\
\text { leptorumol,3,4-dimethoxy cinnamic } \\
\text { acid, 5-hydroxy-2- } \\
\text { methoxybenzaldehyde, p- } \\
\text { methoxybenzoic acid }\end{array}$ & Liver infections & $\begin{array}{l}\text { Zaman et al. 2018, } \\
\text { Mahmood et al. 2013, } \\
\text { Shivhare et al. } 2012\end{array}$ \\
\hline \multirow[t]{3}{*}{ Amaryllidaceae } & Allium cepa L. & Bulb & $\begin{array}{l}\text { Guercetin, fructose, quercetin-3- } \\
\text { glucoside, isorhamnetin-4- } \\
\text { glucoside, xylose, galactose, } \\
\text { glucose, mannose, organosulfur } \\
\text { compounds, allylsulfides, }\end{array}$ & $\begin{array}{l}\text { Bulb is dried and ground powder is used } \\
\text { for cure of jaundice problem }\end{array}$ & $\begin{array}{l}\text { Ullah et al. 2020, } \\
\text { Marrelli et al. } 2019, \\
\text { Medeiros et al. } 2012\end{array}$ \\
\hline & Allium satium $\mathrm{L}$. & Bulb & $\begin{array}{l}\text { Flavonoids, flavenols, S-alk(en)yl } \\
\text { cysteine sulfoxides, cycloalliin, } \\
\text { selenium, thiosulfinates, and sulfu }\end{array}$ & $\begin{array}{l}\text { Bulb is dried and ground powder is used } \\
\text { as hepatodepurative }\end{array}$ & Ceuterick et al. 2008 \\
\hline & Ruscus aculeatus $\mathrm{L}$. & Leaves & $\begin{array}{l}P \text {-coumaric and caffeic acids and } \\
\text { rutin }\end{array}$ & Liver depurative & $\begin{array}{l}\text { Pieroni et al. 2014, } \\
\text { Hadžifejzović et al. } \\
2013\end{array}$ \\
\hline \multirow[t]{2}{*}{ Amaranthaceae } & Achyranthes aspera $\mathrm{L}$ & Root & $\begin{array}{l}\text { Triterpenoid, saponins, alcohol and } \\
\text { 17-penta triacontanol, }\end{array}$ & Used for treatment of jaundice & $\begin{array}{l}\text { Barkatullah, et al. 2015, } \\
\text { Srivastav, } 2011\end{array}$ \\
\hline & $\begin{array}{l}\text { Amaranthus graecizans } \\
\text { subsp. thellungianus } \\
\text { (Nevski) Gusev }\end{array}$ & Aerial parts & Brenan, trolox and gallic acid & Liver tonic & $\begin{array}{l}\text { Yaseen et al. } 2015, \\
\text { Ishtiaq et al. } 2014, \\
\text { Pieroni et al. } 2005\end{array}$ \\
\hline Anacardiaceae & Pistacia integerrima L. & Aerial parts & $\begin{array}{l}\text { Alkaloids, flavonoids, tannins, } \\
\text { saponins and sterols }\end{array}$ & Used for treatment of jaundice & $\begin{array}{l}\text { Jan et al. 2017, Bibi et } \\
\text { al. } 2015\end{array}$ \\
\hline
\end{tabular}




\begin{tabular}{|c|c|c|c|c|c|}
\hline & $\begin{array}{l}\text { Pistacia chinensis Bunge } \\
\text { ssp. integerrima }\end{array}$ & Leaves, bark & $\begin{array}{l}\text { Sitosterol, alnusidiol, gallic acid, } \\
\text { naringenin and amentoflavone }\end{array}$ & $\begin{array}{l}\text { Leaves and bark decoction to cure } \\
\text { jaundice }\end{array}$ & $\begin{array}{l}\text { Ali et al. 2018a, Uddin } \\
\text { et al. } 2011\end{array}$ \\
\hline \multirow[t]{4}{*}{ Apiaceae } & Coriandrum sativum $\mathrm{L}$. & $\begin{array}{l}\text { Leaf, seed, } \\
\text { fruit }\end{array}$ & $\begin{array}{l}\text { Aromatic acid containing } 2- \\
\text { decenoic acid }(30.8 \%) \text {, capric acid } \\
(12.7 \%) \text {, undecanoic acid }(7.1 \%), \\
\text { linalool }(37.7 \%) \text {, grenyl acetate } \\
(17.5 \%), \text { gama-terpinene }(14.4 \%)\end{array}$ & Liver disorders & $\begin{array}{l}\text { Al-Snafi Ali Esmail } \\
2016\end{array}$ \\
\hline & $\begin{array}{l}\text { Pleurospermum candollei } \\
\text { (DC.) Benth. ex C.B. } \\
\text { Clarke }\end{array}$ & Aerial parts & ------ & $\begin{array}{l}\text { A decoction of the whole plant is made } \\
\text { and taken twice a day }\end{array}$ & $\begin{array}{l}\text { Abbas et al. } 2016 \text {, } \\
\text { Agelet et al. } 2003\end{array}$ \\
\hline & $\begin{array}{l}\text { Oenanthe japonica } \\
\text { (Blume) DC. }\end{array}$ & Leaves & $\begin{array}{l}\text { Longiborneol, geranyl linalool and } \\
\text { T-cadinol }\end{array}$ & $\begin{array}{l}\text { Powder of leaves is used for cure of } \\
\text { hepatitis } b\end{array}$ & $\begin{array}{l}\text { Samani \& Kopaei, } \\
2018, \text { Pattram et al. } \\
2011\end{array}$ \\
\hline & $\begin{array}{l}\text { Trachyspermum ammi } \\
\text { (L.) Sprague }\end{array}$ & Leaves & V-terpinene, $\rho$-cymene, and thymol & Used for treatment of hepatitis B & $\begin{array}{l}\text { Samani \& Kopaei 2018, } \\
\text { Gandomi et al. } 2014\end{array}$ \\
\hline Apocynaceae & Carissa spinarum $\mathrm{L}$ & Leaves & $\begin{array}{l}\text { Triterpenes, cardiac glycosides, } \\
\text { sesquiterpenes, monoterpenes, } \\
\text { flavonoids,lignans, fatty acids and } \\
\text { esters }\end{array}$ & Used for treatment of jaundice & $\begin{array}{l}\text { Rashid et al. 2018, } \\
\text { Wangteeraprasert et al. } \\
2012\end{array}$ \\
\hline Aracea & $\begin{array}{l}\text { Colocasia esculenta (L.) } \\
\text { Schott }\end{array}$ & Bulb & $\begin{array}{l}\text { Active phytoconstituents such as } \\
\text { flavonoids, sterols, glycosides, and } \\
\text { other micronutrients }\end{array}$ & $\begin{array}{l}\text { The bulb of this plant cooked as } \\
\text { vegetable And dried bulb is cut in to } \\
\text { pieces and then crushed to make powder } \\
\text { which is used for the treatment of } \\
\text { jaundice }\end{array}$ & $\begin{array}{l}\text { Kidane et al. 2018, } \\
\text { Kumar \& Choyal 2012, }\end{array}$ \\
\hline Asparagaceae & $\begin{array}{l}\text { Asparagus adscendens } \\
\text { Roxb. }\end{array}$ & Root & $\begin{array}{l}\text { Essential oils, asparagine, arginine, } \\
\text { tyrosine, flavonoids (kaempferol, } \\
\text { quercetin, and rutin), resin, and } \\
\text { tannin. }\end{array}$ & $\begin{array}{l}\text { Extract of root is used for treatment of } \\
\text { jaundice }\end{array}$ & $\begin{array}{l}\text { Fawad 2016, Yaseen et } \\
\text { al. 2015, Negi et al. } \\
2010\end{array}$ \\
\hline \multirow[t]{4}{*}{ Asteraceae } & Artemisia campestris L. & Flowers & $\begin{array}{l}\text { B-pinene, spathulenol, } \alpha \text {-pinene, } \\
\text { limonene and o-cymene }\end{array}$ & Hepatic protector & $\begin{array}{l}\text { Al-Jahid et al. 2017, } \\
\text { Asadi et al. } 2015\end{array}$ \\
\hline & Artemisia genipi Stechm. & Whole plant & Sesquiterpene lactones & $\begin{array}{l}\text { For the treatment of inflammation of } \\
\text { jaundice }\end{array}$ & $\begin{array}{l}\text { Vitalini et al. 2013, } \\
\text { Appendino et al. } 1982\end{array}$ \\
\hline & Ageratum conyzoides & Leaves & $\begin{array}{l}\text { Alkaloids, flavonoids, tannins, } \\
\text { saponins, and cyanic acid }\end{array}$ & $\begin{array}{l}\text { Lease paste is used for treatment of } \\
\text { Jaundice }\end{array}$ & $\begin{array}{l}\text { Zaman et al. 2018, } \\
\text { Okunade } 2002\end{array}$ \\
\hline & $\begin{array}{l}\text { Carthamus oxyacantha } \\
\text { M.Bieb }\end{array}$ & Flowers & $\begin{array}{l}\text { Linoleic oil, palmitic acid, stearic } \\
\text { acid, oleic acid, linoleic acid }\end{array}$ & Flowers are used for the cure of Jaundice & $\begin{array}{l}\text { Zaman et al. 2018, } \\
\text { Ahmad et al. 2009, } \\
\text { Ahmad et al. } 2007\end{array}$ \\
\hline
\end{tabular}




\begin{tabular}{|c|c|c|c|c|c|}
\hline & Calendula officinalis $\mathrm{L}$. & Flowers & $\begin{array}{l}\text { Flavonoids, particularly patulitrin } \\
\text { and patuletin }\end{array}$ & Infusion is used to cure liver diseases & $\begin{array}{l}\text { Pieroni et al. 2012, } \\
\text { Mulley et al. } 2009\end{array}$ \\
\hline & Cynara scolymus $L$. & $\begin{array}{l}\text { Leaves, } \\
\text { flowers }\end{array}$ & $\begin{array}{l}\text { Cynarin, cynaroside, scolymoside, } \\
\text { ferulic and caffeoylquinic }\end{array}$ & Leaves and flowers hepatitis & $\begin{array}{l}\text { Samani \& Kopaei 2018, } \\
\text { Ceuterick et al. 2011, } \\
\text { Pieroni et al. 2004, Lutz } \\
\text { et al. } 2011\end{array}$ \\
\hline & Eclipta prostrata (L.) L & Aerial parts & $\begin{array}{l}\text { Glycosides eclalbasaponin I and } \\
\text { eclalbasaponin II }\end{array}$ & $\begin{array}{l}\text { Decoction of boiled whole parts is used for } \\
\text { the treatment of Jaundice, hepatitis and } \\
\text { other liver disorders }\end{array}$ & $\begin{array}{l}\text { Zaman et al. } \\
\text { 2018,Shinwar et al. 2016, } \\
\text { Mahmood et al. } 2012\end{array}$ \\
\hline & $\begin{array}{l}\text { Tanacetum artemisioides } \\
\text { L. }\end{array}$ & Aerial parts & $\begin{array}{l}\text { Flavonoids, tanacetamide,5- } \\
\text { demethylnobiletin }\end{array}$ & To cure hepatitis B & $\begin{array}{l}\text { Hussain et al. 2018, } \\
\text { Hussain et al. 2013 }\end{array}$ \\
\hline & Taraxicum officinale L. & Leaves & $\begin{array}{l}\text { Proteins, fatty acid, potassium, } \\
\text { calcium }\end{array}$ & $\begin{array}{l}\text { For the treatment of inflammation of } \\
\text { jaundice }\end{array}$ & $\begin{array}{l}\text { Samani \& Kopaei 2018, } \\
\text { Jan et al. 2017, Pieroni et } \\
\text { al. 2014, Hussain et al. } \\
\text { 2012, Ikram \& } \\
\text { Hussain1978, Escudero } \\
\text { et al. } 2003\end{array}$ \\
\hline & Silybum marianum L. & $\begin{array}{l}\text { Leaf,fruit, } \\
\text { seed }\end{array}$ & $\begin{array}{l}\text { Flavoligrans, silybin, isosiybin and } \\
\text { silymarin (6\%), Flavolagnans, } \\
\text { silybin, silydianin, silychristine }\end{array}$ & To cure jaundice and hepatitis & $\begin{array}{l}\text { Samani \& Kopaei, } \\
\text { 2018,Khan et al. 2016, } \\
\text { Foster } 1991\end{array}$ \\
\hline & Sonchus asper (L.) Hill & Aerial parts & $\begin{array}{l}\mathrm{N}-\text { Hexane, alkaloids, saponins, } \\
\text { flavonoids, phenols and tannins }\end{array}$ & To cure hepatitis B & $\begin{array}{l}\text { Ali et al. 2020, } \\
\text { Hussain et al. } 2018\end{array}$ \\
\hline Berberidaceae & Berberis lycium Royle. & Fruit, root & $\begin{array}{l}\text { Alkaloids, cardioactive glycosides, } \\
\text { saponins, tannins, anthocyanins, } \\
\text { vitamins, carbohydrates, proteins, } \\
\text { lipids, fiber content, } \beta \text { carotein, } \\
\text { cellulose, phytic acid and phytate } \\
\text { phosphorous. }\end{array}$ & For the treatment of jaundice & $\begin{array}{l}\text { Ali et al. 2018b, Rashid } \\
\text { et al. 2018, Ahmad et } \\
\text { al. } 2017 \text { Shabir et al. } \\
\text { 2012, Tyler et al. } 1981\end{array}$ \\
\hline
\end{tabular}




\begin{tabular}{|c|c|c|c|c|c|}
\hline & $\begin{array}{l}\text { Berberis pseudumbellata } \\
\text { R. Parker }\end{array}$ & $\begin{array}{l}\text { Seeds,flower } \\
\text {, fruits, }\end{array}$ & $\begin{array}{l}\text { Alkaloids, steroids, glycosides, } \\
\text { flavonoids, saponins, terpenoids } \\
\text { and reducing sugar }\end{array}$ & $\begin{array}{l}\text { Seeds and fresh fruits are eaten while a } \\
\text { flower decoction is recommended three } \\
\text { times a day }\end{array}$ & $\begin{array}{l}\text { Yasil \& Inal 2019, Khan } \\
\text { et al. } 2016\end{array}$ \\
\hline Boraginaceae & $\begin{array}{l}\text { Onosma hispida Wall. \& } \\
\text { G. Don. }\end{array}$ & Aerial parts & $\begin{array}{l}\text { Naphthaquinones, alkaloids and } \\
\text { phenolic compounds }\end{array}$ & $\begin{array}{l}\text { The whole plant is cooked in water as a } \\
\text { vegetable and taken twice a day as } \\
\text { needed }\end{array}$ & $\begin{array}{l}\text { Yeşil \& İnal 2019, } \\
\text { Kumar et al. } 2012\end{array}$ \\
\hline \multirow[t]{3}{*}{ Brassicaceae } & Brassica rapa L. & Flowers & $\begin{array}{l}\beta \text {-sitosteryl-3} \beta \text {-glucopyranoside- } 6 \text { '- } \\
\text { O-fatty acid esters, } \beta \text {-sitosterol, } \\
\text { chlorophyll and phytyl fatty acid } \\
\text { esters }\end{array}$ & Used for treatment of hepatitis & $\begin{array}{l}\text { Ragasa et al. 2016, } \\
\text { Barkatullah, et al. } 2015\end{array}$ \\
\hline & Lepidium sativum $L$. & Leaves & $\begin{array}{l}\text { Hexadecadienoic acid, } \\
\text { heneicosanoic acid,10- } \\
\text { octadecenoic acid,15-tetracosenoic } \\
\text { acid, hexadecanoic acid and steric } \\
\text { acid }\end{array}$ & Leaves powder is used to treat hepatitis & $\begin{array}{l}\text { Samani \& Kopaei, } \\
\text { 2018, } \\
\text { Gokavi et al. } 2004\end{array}$ \\
\hline & Rhaphanus sativus $\mathrm{L}$. & Root & $\begin{array}{l}\text { Raphanin, glycosinolates, } \\
\text { enzymes, trace elements, } \\
\text { anthocyanin, pectin, protein, and } \\
\text { Vitamin C }\end{array}$ & $\begin{array}{l}\text { Decoctions of its roots are recommended } \\
\text { for the treatment of jaundice. Fleshy part } \\
\text { and its juice is used for treatment of } \\
\text { jaundice }\end{array}$ & $\begin{array}{l}\text { Ong \& Kim 2014, } \\
\text { Prajapati et al. } 2006 \text {, }\end{array}$ \\
\hline Burseraceae & Boswellia carterii Birdw. & Stem (resin) & $\begin{array}{l}\text { Essential oils, pyrolysates boswellic } \\
\text { acids }\end{array}$ & Resin is used for cure of hepatitis C & $\begin{array}{l}\text { Samani \& Kopaei 2018, } \\
\text { Moussaieff et al. } 2008\end{array}$ \\
\hline Caryophyllaceae & Herniaria glabra L. & Leaves & $\begin{array}{l}\text { Triterpene,saponins:herniaria } \\
\text { saponins, gypsogen, flavonoids and } \\
\text { hydroxycoumarins }\end{array}$ & For jaundice (anti-icterus) & $\begin{array}{l}\text { Alsanfi 2018, Rigat et } \\
\text { al. } 2007\end{array}$ \\
\hline \multirow[t]{2}{*}{ Chenopodiaceae } & Chenopodium album L. & Aerial parts & $\begin{array}{l}\text { Phenols, tannins, phytic acid } \\
\text { phytate phosphorus, alkaloids }\end{array}$ & For the cure of hepatic disorders & $\begin{array}{l}\text { Zaman et al. 2018, Jan } \\
\text { et al. 2017, Alsanif } \\
2015, \text { Ceuterick et al. } \\
2008\end{array}$ \\
\hline & $\begin{array}{l}\text { Chenopodium } \\
\text { ambrosioides L. }\end{array}$ & $\begin{array}{l}\text { Leaves, } \\
\text { flowers }\end{array}$ & $\begin{array}{l}\text { Cymene, alpha-terpinene and } \\
\text { limonene }\end{array}$ & Hepatodepurative & $\begin{array}{l}\text { Ceuterick et al. 2011, } \\
\text { Barros et al. } 2013\end{array}$ \\
\hline Convolvulaceae & $\begin{array}{l}\text { Ipomoea paniculata Burm. } \\
\text { f }\end{array}$ & Aerial parts & $\begin{array}{l}\text { Tetracosane, myristic acid, beta- } \\
\text { sitosterol, beta-carotene, } \\
\text { daucosterol and quercetin }\end{array}$ & Liver cirrhosis & $\begin{array}{l}\text { Ahmad et al. 2018, } \\
\text { Heiurich et al. } 1998\end{array}$ \\
\hline Crassulaceae & $\begin{array}{l}\text { Rhodiola kirilowii (Regel) } \\
\text { Maxim. }\end{array}$ & Root & $\begin{array}{l}\text { Arbutin, epigallocatechin gallate, } \\
\text { rhodiocaynocide, lotaustralin }\end{array}$ & Root powder is used to cure hepatitis C & $\begin{array}{l}\text { Samani \& Kopaei 2018, } \\
\text { Wiedenfeld et al. } 2007\end{array}$ \\
\hline
\end{tabular}




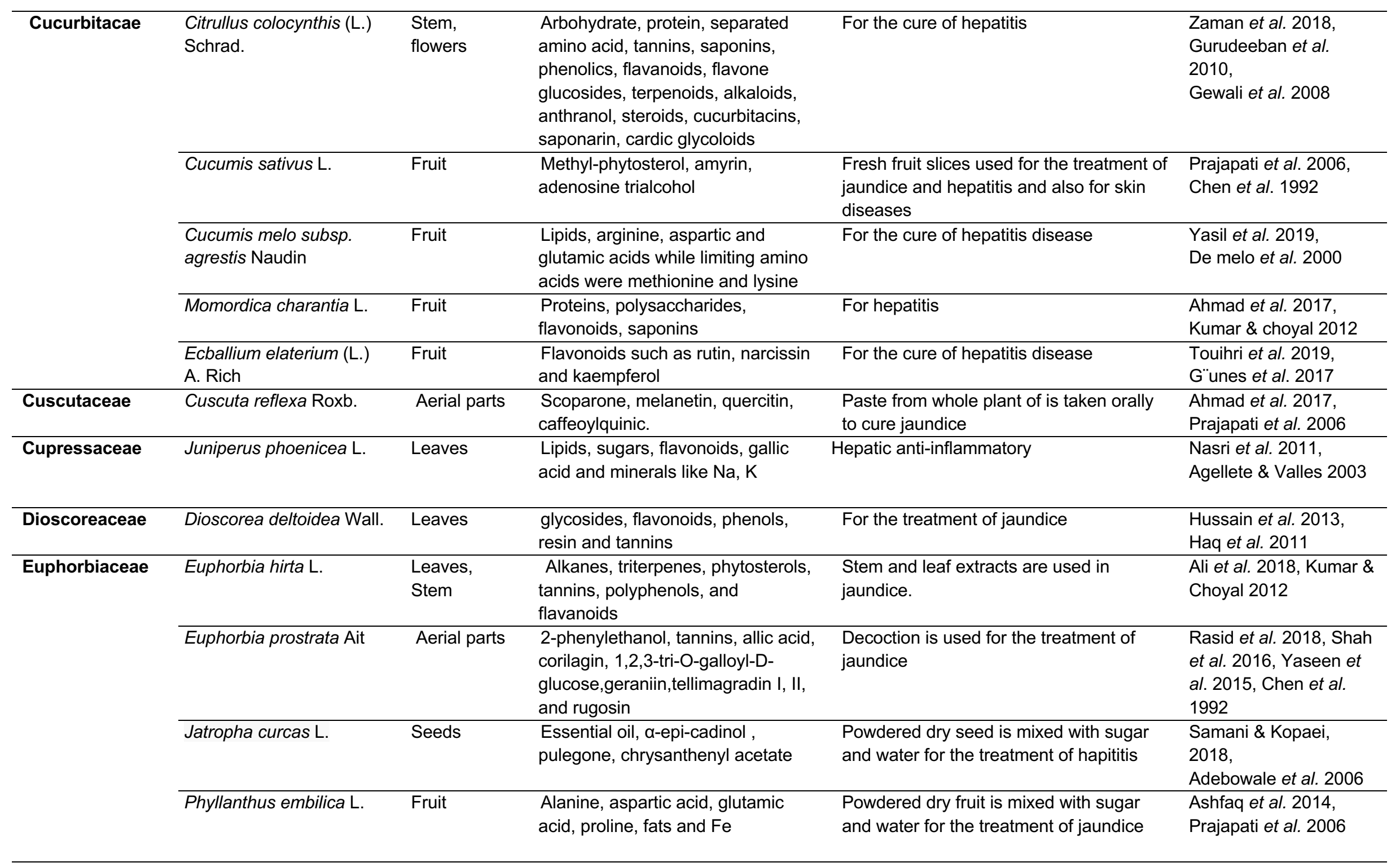




\begin{tabular}{|c|c|c|c|c|c|}
\hline & Phyllanthus niruri L. & Root & $\begin{array}{l}\text { Phyllanthine, flavonoids, quercetin, } \\
\text { isoquercetin, }\end{array}$ & Liver diseases & $\begin{array}{l}\text { Farooq et al. 2019, } \\
\text { Prajapati et al. } 2006\end{array}$ \\
\hline Equisetaceae & $\begin{array}{l}\text { Hippochaete debilis } \\
\text { (Roxb. ex Vaucher) }\end{array}$ & Aerial parts & $\begin{array}{l}\text { Ash, Lipid, protein, Ca, Ascorbic } \\
\text { acid, and niacin }\end{array}$ & For hepatitis & $\begin{array}{l}\text { Tareen et al. 2010, } \\
\text { Hakim et al. 2008, } \\
\text { Mangario et al. } 1995\end{array}$ \\
\hline \multirow{5}{*}{ Fabaceae } & $\begin{array}{l}\text { Acacia jacquemontii } \\
\text { Benth }\end{array}$ & Leaves & $\begin{array}{l}\text { Ester-cetyl } \\
\text { triacontanoate along with n- } \\
\text { triacontanol, n-octacosanol, B- } \\
\text { sitosterol and stigmasterol }\end{array}$ & Extract of leaves is used for jaundice & $\begin{array}{l}\text { Yaseen et al. 2015, } \\
\text { Singh et al. } 2010\end{array}$ \\
\hline & $\begin{array}{l}\text { Argyrolobium roseum } \\
\text { (Cambess.) Jaub. \& } \\
\text { Spach }\end{array}$ & Aerial parts & $\begin{array}{l}\text { Favonoids, glycoside, and vitexin, } \\
\text { D-pinitol }\end{array}$ & Used for liver diseases & $\begin{array}{l}\text { Ram et al. 2007, Gupta } \\
\text { et al. 2005, }\end{array}$ \\
\hline & Cassia fistula $\mathrm{L}$. & $\begin{array}{l}\text { Leaaves, } \\
\text { flower }\end{array}$ & $\begin{array}{l}\text { Gum containing arabin, tannic and } \\
\text { galic acid }\end{array}$ & Hepatitis & $\begin{array}{l}\text { lqbal et al. 2016, } \\
\text { Adnan et al. 2014 }\end{array}$ \\
\hline & Glycyrrhiza glabra L. & Root & $\begin{array}{l}\text { Glycyrrhitic acid,multiple flavonoids, } \\
\text { isoflavonoids, sterols }\end{array}$ & Hepatitis & $\begin{array}{l}\text { Mahmood et al. 2013, } \\
\text { Dhiman et al. } 2005\end{array}$ \\
\hline & Tamarindus indica L. & Root, fruit & $\begin{array}{l}\beta \text {-amyrin, compesterol, } \beta \text {-sitosterol } \\
\text { and seven hydrocarbons. The aerial } \\
\text { parts of this plant have } \\
\text { demonstrated the presence of } \\
\text { tartaric acid, acetic acid, and } \\
\text { succinic acid, gum, pectin, sugar, } \\
\text { tannins, alkaloid, flavonoids, } \\
\text { sesquiterpenes, and glycosides. }\end{array}$ & $\begin{array}{l}\text { A decoction of its roots is used in the } \\
\text { treatment of Jaundice, hepatitis and other } \\
\text { liver disorders. Fruit also act as a remedy } \\
\text { for the treatment of jaundice }\end{array}$ & $\begin{array}{l}\text { Kidane et al. 2018, } \\
\text { Kumar \& Choyal, } 2012\end{array}$ \\
\hline
\end{tabular}




\begin{tabular}{|c|c|c|c|c|c|}
\hline & $\begin{array}{l}\text { Trigonella foenum } \\
\text { graecum (Nevski) Afan. \& } \\
\text { Gontsch. }\end{array}$ & Seeds & $\begin{array}{l}\mathrm{N}, \mathrm{N} \text { '-dicarbazyl, glycerol } \\
\text { monopalmitate, stearic acid, beta- } \\
\text { sitosteryl glucopyranoside, ethyl- } \\
\text { alpha-D-glucopyranoside, D-3-O- } \\
\text { methyl-chiroinsitol and sucrose }\end{array}$ & Seeds are used to cure hepatitis & $\begin{array}{l}\text { Samani \& Kopaei 2018, } \\
\text { Shang et al. } 2002\end{array}$ \\
\hline & Sophora flavescens Aiton & Root & $\begin{array}{l}\text { Maackiain; trifolirhizin 6'- } \\
\text { monoacetate, formononetin,2,4- } \\
\text { dihydroxy benzoic acid and \#beta\#- } \\
\text { sitosterol }\end{array}$ & $\begin{array}{l}\text { Root decoction is used for the cure of } \\
\text { hepatitis C }\end{array}$ & $\begin{array}{l}\text { Samani \& Kopaei 2018, } \\
\text { Li et al. } 2004\end{array}$ \\
\hline Fagaceae & $\begin{array}{l}\text { Quercus infectoria G. } \\
\text { Olivier }\end{array}$ & Seeds & Syringic, gallic and ellagic acids & $\begin{array}{l}\text { Seeds are rusted in hot ash used for the } \\
\text { control of hepatitis C }\end{array}$ & $\begin{array}{l}\text { Samani \& Kopaei 2018, } \\
\text { Ikram \& Nowshad, } \\
1977\end{array}$ \\
\hline \multirow[t]{2}{*}{ Fumaraceae } & $\begin{array}{l}\text { Fumaria indica (Hausskn.) } \\
\text { Pugsley }\end{array}$ & $\begin{array}{l}\text { Leaves, } \\
\text { flowers }\end{array}$ & $\begin{array}{l}\text { Alkaloids, oxyhydrastinine, } \\
\text { noroxyhydrastinine, fumaramine, } \\
\text { stylopine, bisnorargemonine and } \\
\text { fumaritine }\end{array}$ & $\begin{array}{l}\text { Mixture powder of leaves and flowers } \\
\text { used for cure of hepatitis }\end{array}$ & $\begin{array}{l}\text { Shah et al. 2016; P } \\
\text { Gupta et al. } 2012 \text { Asia }\end{array}$ \\
\hline & Fumaria officinalis $\mathrm{L}$ & Aerial parts & $\begin{array}{l}\text { Allocryptopine, chelidonine, } \\
\text { protopine, bicuculline, sanguinarine, } \\
\text { cheleritrine, stylopine and } \\
\text { hydrastine }\end{array}$ & For the treatment of jaundice & $\begin{array}{l}\text { Ahmad et al. } 2017, \\
\text { Paltinean et al. } 2016, \\
\text { Bhatia et al. } 2014\end{array}$ \\
\hline Ganodermataceae & $\begin{array}{l}\text { Ganoderma lucidum } \\
\text { (Curtis) P. Karst }\end{array}$ & Fruiting body & Triterpenoids and polysaccharides & $\begin{array}{l}\text { Fruiting body cooked and useful for } \\
\text { hepatitis B }\end{array}$ & $\begin{array}{l}\text { Samani \& Kopaei, } \\
2018, \\
\text { Boh et al. } 2007\end{array}$ \\
\hline \multirow[t]{4}{*}{ Gentianaceae } & $\begin{array}{l}\text { Centaurium erythraea } \\
\text { Rafin. }\end{array}$ & Leaves & $\begin{array}{l}\text { Loganin, copaene, selinene, and } \\
\text { alpha-cadinol }\end{array}$ & For the treatment of jaundice & $\begin{array}{l}\text { Mustafa et al. 2012, } \\
\text { Yöney et al. } 2010\end{array}$ \\
\hline & Swertia petiolata D. Don & Aerial parts & $\begin{array}{l}\text { Flavonoids, steroids, triterpenoids, } \\
\text { alkaloids, glycosides, } \\
\text { carbohydrates, tannins, phenolic } \\
\text { compounds e }\end{array}$ & For the treatment of jaundice & $\begin{array}{l}\text { Samani \& Kopaei, } \\
\text { 2018, Ahmad et al. } \\
\text { 2017, Bader et al. } 2017\end{array}$ \\
\hline & $\begin{array}{l}\text { Swertia chirayita (Roxb.) } \\
\text { Buch.-Ham. ex C.B. } \\
\text { Clarke }\end{array}$ & Leaves & $\begin{array}{l}\text { Stigmasterol } 4 \text { and 1,5-dihydroxy-3- } \\
\text { methoxyxanthone-8-O- } \beta \text {-D- } \\
\text { glucopyranoside }\end{array}$ & For the treatment of hepatitis $B$ & $\begin{array}{l}\text { Samani \& Kopaei 2018, } \\
\text { Negi et al. } 2011\end{array}$ \\
\hline & Swertia patens Burkill & Aerial parts & $\begin{array}{l}\text { Elucidated, angelone, gentiogena, } \\
\text { erythricin, erythrocentaurin, } \\
\text { gentianine, swertiakoside, } \\
\text { swertiamarin, actylswertiamarin, } \\
\text { and amarogentin }\end{array}$ & For the cure of hepatitis B & $\begin{array}{l}\text { Samani \& Kopaei 2018, } \\
\text { He et al., } 2015\end{array}$ \\
\hline
\end{tabular}




\begin{tabular}{|c|c|c|c|c|c|}
\hline Hippocastinaceae & $\begin{array}{l}\text { Aesculus indica (Wall. ex. } \\
\text { Cambl.) Hook.f. }\end{array}$ & Seeds & $\begin{array}{l}\text { Flavonols, phenols ree } \alpha \text {-amino } \\
\text { acids and carbohydrates }\end{array}$ & Used for the treatment of jaundice & $\begin{array}{l}\text { Paterska et al. 2017, } \\
\text { Haq et al. } 2011\end{array}$ \\
\hline Hypericaceae & Hypericum perforatum L. & Aerial parts & $\begin{array}{l}\text { Flavonoids, rutin, hyperoside, } \\
\text { isoquercetin, quercitrin, quercetin, } \\
\text { amentoflavone, biapigenin, astilbin, } \\
\text { myricetin, miquelianin, kaempferol, } \\
\text { luteolin, phenolic acids }\end{array}$ & Infusion is used for liver diseases & $\begin{array}{l}\text { Pieroni et al. 2012,m } \\
\text { Egea et al. 2015, } \\
\text { Nahrstedt et al. } 1997\end{array}$ \\
\hline \multirow[t]{5}{*}{ Lamiaceae } & $\begin{array}{l}\text { Ajuga bracteosa Wall. ex } \\
\text { Benth }\end{array}$ & Leaves & $\begin{array}{l}\text { Exacosanol, B-sitosterol, } \\
\text { tetracosanoic acid and B-sitosterol- } \\
\text { B-D-glucoside }\end{array}$ & Used for liver jaundice & $\begin{array}{l}\text { Ali et al. 2018, Haq et } \\
\text { al. 2011, Riaz et al. } \\
2007\end{array}$ \\
\hline & Mentha longifolia (L.) L & Leaves & $\begin{array}{l}\text { Pulegone, isomenthone, } 1,8- \\
\text { cineole, borneol and menthol }\end{array}$ & Used for the treatment of hepatitis $A$ & $\begin{array}{l}\text { Samani \& Kopaei } 2018 \text {, } \\
\text { Mkaddem et al. } 2009\end{array}$ \\
\hline & Ocimum basilicum L. & $\begin{array}{l}\text { Leaves, } \\
\text { flowers tops }\end{array}$ & Eugenol and linalool & & $\begin{array}{l}\text { Samani \& Kopaei 2018, } \\
\text { Govindarajan et al. } \\
2013\end{array}$ \\
\hline & Salvia bucharica M. Pop & Flowers & $\begin{array}{l}\text { Terpenoids, bucharioside and } \\
\text { buchariol }\end{array}$ & Infusion of flowers are useful in jaundice & $\begin{array}{l}\text { Bibi et al. 2014, Ahmad } \\
\text { et al. } 1999\end{array}$ \\
\hline & Rosmarinus officinalis L & Leaves & $\begin{array}{l}\text { Phenolic compounds, di- and } \\
\text { triterpenes and essential oils }\end{array}$ & $\begin{array}{l}\text { Leaves pase is used for the treatment of } \\
\text { jaundice }\end{array}$ & $\begin{array}{l}\text { Idm'hand et al. } 2020 \text {, } \\
\text { Andrade et al. } 2018\end{array}$ \\
\hline Lythraceae & $\begin{array}{l}\text { Lagerstroemia speciosa } \\
\text { (L.) Pers }\end{array}$ & $\begin{array}{l}\text { Leaves, } \\
\text { flowers }\end{array}$ & $\begin{array}{l}\text { Corosoli acid }{ }^{6} \text {, lageracetal, amyl } \\
\text { alcohol, ellagic acid, gallic acid, 4- } \\
\text { hydroxyl benzoic acid, beta } \\
\text { sitosterol, Asiatic acid, alphitolic } \\
\text { acid }\end{array}$ & For the treatment of jaundice & $\begin{array}{l}\text { Ong \& Kim 2014, Bai et } \\
\text { al. } 2008\end{array}$ \\
\hline \multirow[t]{3}{*}{ Malvaceae } & Abutilon indicum $\mathrm{L}$. & $\begin{array}{l}\text { Leaves, } \\
\text { flowers }\end{array}$ & $\begin{array}{l}\text { Carbohydrates, steroids, } \\
\text { glycosides, flavonoids, tannins and } \\
\text { Phenolic compounds }\end{array}$ & For cure of liver diseases & $\begin{array}{l}\text { Yaseen et al. 2015, } \\
\text { Kuo et al. } 2008\end{array}$ \\
\hline & Alcea rosea $\mathrm{L}$ & Whole plant & $\begin{array}{l}\text { Flavonoid, salicylic, vanillic, ferulic, } \\
\text { syringic, caffeic, p-hydroxybenzoic, } \\
\text { p-coumaric, and p- } \\
\text { hydroxyphenylacetic acid }\end{array}$ & $\begin{array}{l}\text { Whole plant is powder and decoction is } \\
\text { used for jaundice }\end{array}$ & $\begin{array}{l}\text { Ullah et al. 2020, } \\
\text { Ammar et al. } 2013\end{array}$ \\
\hline & $\begin{array}{l}\text { Corchorus depressus (L.) } \\
\text { Stock }\end{array}$ & Aerial parts & $\begin{array}{l}\text { Flavonol } \\
\text { glycosides, } \\
\text { flavonoids, } \\
\text { steroids }\end{array}$ & Liver tonic & $\begin{array}{l}\text { Yaseen et al. 2015, } \\
\text { Zahid et al. } 2002\end{array}$ \\
\hline
\end{tabular}




\begin{tabular}{|c|c|c|c|c|c|}
\hline Meliaceae & Melia azedarach L. & Leaves & $\begin{array}{l}\text { Quercetin, astragalin and 2- } \\
\text { methoxy-4-(2-propenyl)phenyl } \beta \text {-D- } \\
\text { glucoside }\end{array}$ & Used for the treatment of jaundice & $\begin{array}{l}\text { Bibi et al. 2014, Kumar } \\
\text { \& Choyal 2012, } \\
\text { Hadjiakhoondi et al. } \\
2006\end{array}$ \\
\hline \multirow[t]{3}{*}{ Menispermaceae } & $\begin{array}{l}\text { Stephania abyssinica } \\
\text { Dillon and A. Rich }\end{array}$ & $\begin{array}{l}\text { Leaves, } \\
\text { flowers }\end{array}$ & $\begin{array}{l}\text { Oxoxylopine, liriodenine, } \\
\text { atherospermidine, }\end{array}$ & $\begin{array}{l}\text { The leaf part is boiled, and about } 1 \text { cup is } \\
\text { drunk for liver disease }\end{array}$ & $\begin{array}{l}\text { Tuasha et al. 2018, } \\
\text { Omole et al. } 2014\end{array}$ \\
\hline & $\begin{array}{l}\text { Tinospora cordifolia } \\
\text { (Willd) Miers. }\end{array}$ & Stem & $\begin{array}{l}\text { Terpenoid, alkaloid, lignans, } \\
\text { steroids. }\end{array}$ & $\begin{array}{l}\text { Fresh stem decotion is considered good } \\
\text { for the treatment of jaundice and seminal } \\
\text { weakness. }\end{array}$ & Kumar \& Choyal 2012 \\
\hline & Peumus boldus Molina & Leaves & $\begin{array}{l}\text { Trans-sabinene, pinocarveol, } \\
\text { pinocarvone, 4-terpineol, } \\
\text { ascaridole, piperitone oxide, and } \\
\text { limonene dioxide }\end{array}$ & $\begin{array}{l}\text { Hepatodepurative, liver ailments, } \\
\text { combined with lemon juice, to balance } \\
\text { the harmful properties should not be } \\
\text { taken during more than one week, } 3 \\
\text { leaves/cup, } 3 \text { times/day }\end{array}$ & $\begin{array}{l}\text { Ceuterick et al. 2011, } \\
\text { Ceuterick et al. 2008, } \\
\text { Mazutti et al. } 2008\end{array}$ \\
\hline \multirow[t]{2}{*}{ Moraceae } & Morus alba L. & Fruit & $\begin{array}{l}\text { Ascorbic acid, carotene, vitamin D, } \\
\text { glutathione, } \mathrm{Ca}, \mathrm{Cu}, \mathrm{Zn} \text {, Volatile oil, } \\
\text { nicotinic acid }\end{array}$ & $\begin{array}{l}\text { Crushed fruits are used to cure jaundice } \\
\text { and hepatitis }\end{array}$ & $\begin{array}{l}\text { Zaman et al. 2018, } \\
\text { Bhatia et al. 2014, } \\
\text { Pieroni 2008a, Haq \& } \\
\text { Hussain et al. 2007, } \\
\text { Ercisli \& Orhan } 2007\end{array}$ \\
\hline & Morus nigra L. & Fruit & $\begin{array}{l}\text { Olcanolic acid, apigenin, } \\
\text { cyclocommunol., morusin and } \\
\text { cyclomorusin }\end{array}$ & To cure jaundice & $\begin{array}{l}\text { Wang et al. 2007, } \\
\text { Kassoye et al. } 2006\end{array}$ \\
\hline Myrsinaceae & Embelia schimperi Vatke & Fruit & $\begin{array}{l}\text { Alkaloid benzoate, oxacillin, } \\
\text { embelin and tetracycline }\end{array}$ & Fruit is used to cure hepatitis C & $\begin{array}{l}\text { Samani \& Kopaei 2018, } \\
\text { Van Damme \& Kokoska } \\
2015\end{array}$ \\
\hline Nyctaginaceae & Boerhavia diffusa $L$. & Aerial parts & $\begin{array}{l}\text { B-Sitosterol, a-2-sitosterol, palmitic } \\
\text { acid, ester of b-sitosterol, } \\
\text { tetracosanoic, hexacosonoic, } \\
\text { stearic, arachidic acid, urosilic acid, } \\
\text { Hentriacontane, b-ecdysone, } \\
\text { triacontanol }\end{array}$ & Used to treat of jaundice & $\begin{array}{l}\text { Malede et al. } 2020 \\
\text { Maity et al. } 2015\end{array}$ \\
\hline Myrtaceae & $\begin{array}{l}\text { Syzygium aromaticum (L.) } \\
\text { Merr. \& L.M. Perry }\end{array}$ & Flower bud & $\begin{array}{l}\text { Kaempferol, myricetin, } \\
\text { rhamnocitrin, gallic acid, oleanolic } \\
\text { acid, ellagic acid, and flavonoids }\end{array}$ & Used to treat of hepatitis C & $\begin{array}{l}\text { Batiha et al. 2020, } \\
\text { Samani \& Kopaei } 2018\end{array}$ \\
\hline Oleaceae & Olea europaea L & Aerial parts & $\begin{array}{l}\text { Caffeic acid, verbascoside, } \\
\text { oleuropein, luteolin } 7-O \text {-glucoside, } \\
\text { rutin and apigenin }\end{array}$ & Hepatoprotective & $\begin{array}{l}\text { Pieroni et al. 2014, } \\
\text { Periera et al. } 2007\end{array}$ \\
\hline
\end{tabular}




\begin{tabular}{|c|c|c|c|c|c|}
\hline Oxalidaceae & Oxalis corniculata L. & Leaves & $\begin{array}{l}\text { Flavonoids, vitamin } C \text { and } \beta- \\
\text { carotene, minerals like calcium, } \\
\text { potassium }\end{array}$ & For the cure of hepatic disorders & $\begin{array}{l}\text { Jan et al. 2017,Srikanth } \\
\text { et al. } 2012\end{array}$ \\
\hline Papaveraceae & Papaver somniferum L. & Fruit & $\begin{array}{l}\text { Codeine, morphine, noscapine, } \\
\text { papaverine, and thebaine }\end{array}$ & Used for liver diseases & $\begin{array}{l}\text { Ullah et al. 2020, Da } \\
\text { Cheng et al. } 2015\end{array}$ \\
\hline Phyllanthaceae & Phyllanthus emblica L. & Fruit & Flavonoids & For cure of jaundice & $\begin{array}{l}\text { Habib-ur-Rehman } \\
2007, \\
\text { Kadir et al. } 2007\end{array}$ \\
\hline Piperaceae & Piper cubeba Bojer & Root & $\begin{array}{l}\text { Terpinen-4-ol (42.41\%), } \alpha \text {-copaene } \\
(20.04 \%) \text {, and } \gamma \text {-elemene }\end{array}$ & For the cure of hepatitis B & $\begin{array}{l}\text { Rigat et al. 2007, Blick } \\
1996\end{array}$ \\
\hline \multirow[t]{3}{*}{ Poaceae } & $\begin{array}{l}\text { Desmostachya bipinnata } \\
\text { (L.) Stap }\end{array}$ & Root & $\begin{array}{l}\text { Camphene, isobornyl acetate, } \\
\text { caryophyllene diepoxide, } \beta \text { - } \\
\text { eudesmol, eseroline and calarene }\end{array}$ & $\begin{array}{l}\text { Juice of the root about } 4 \text { teaspoons three } \\
\text { times a day is given in case of jaundice. }\end{array}$ & $\begin{array}{l}\text { Malla et al. } 2015, \\
\text { Kumar et al. } 2010\end{array}$ \\
\hline & Hordeum vulgare $\mathrm{L}$. & Seed & $\begin{array}{l}\text { Arginine, Histidine, lysine, tyrosine, } \\
\text { methionine }\end{array}$ & Dried seeds are used for hepatitis & Prajapati et al. 2006 \\
\hline & Saccharum officinarum L. & Stem & $\begin{array}{l}\text { Flavonoids:naringenin, tricin, } \\
\text { apigenin and luteolin derivatives }\end{array}$ & $\begin{array}{l}\text { Juice extracted from stem considered } \\
\text { good remedy for jaundice }\end{array}$ & Kumar \& Choyal 2012 \\
\hline Portulacaceae & Portulaca quadrifida L. & Leaves & $\begin{array}{l}\text { Gallotannins, omega- } 3 \text { fatty acids, } \\
\text { ascorbic acid, } \alpha \text {-tocopherols, } \\
\text { kaempferol, quercetin, and apigenin }\end{array}$ & For cure of hepatic & $\begin{array}{l}\text { Zaman et al. 2018, } \\
\text { Zhou et al. } 2015\end{array}$ \\
\hline Podocarpaceae & $\begin{array}{l}\text { Podocarpus falcatus } \\
\text { (Thunb.) Mirb }\end{array}$ & Stem, leaves & $\begin{array}{l}\text { Nagilactones, dilactones and } \\
\text { derivates }\end{array}$ & $\begin{array}{l}\text { The shoot is boiled, and } 1 \text { cup is drunk; } \\
\text { Oozing liquid from the stem is mixed with } \\
\text { cold water and is drunk for cure of liver } \\
\text { disease }\end{array}$ & $\begin{array}{l}\text { Tuasha et al., 2018, } \\
\text { Addo et al., } 2015\end{array}$ \\
\hline \multirow[t]{2}{*}{ Polygonaceae } & Rumex hastatus D. Don & Aerial parts & $\begin{array}{l}\text { Triterpenoids, stilbene glycosides, } \\
\text { tannic acid, saponins, resveratrol, } \\
\text { sterols, amino acids, alkaloids, } \\
\text { phenolic compunds and vitamin }\end{array}$ & For treatment of jaundice & $\begin{array}{l}\text { Haq et al. 2011, Liang } \\
\text { et al. } 2010, \text { Pieroni et } \\
\text { al. } 2008\end{array}$ \\
\hline & $\begin{array}{l}\text { Rumex nepalensis } \\
\text { Spreng. }\end{array}$ & Aerial parts & $\begin{array}{l}\text { Torachrysone, rumexoside, } \\
\text { orientaloside, orcinol glucoside, } \\
\text { aloesin and lyoniresinol }\end{array}$ & For the treatment of liver disease & $\begin{array}{l}\text { Liang et al. 2010, Giday } \\
\text { et al. 2009, }\end{array}$ \\
\hline Punicaeae & Punica granatum L. & Fruit,seed & $\begin{array}{l}\text { Citric acid, sorbitol, mannitol, } \\
\text { pelletierine, isoquercetin, glucose, } \\
\text { sucrose, maltose, and oxalic acid }\end{array}$ & $\begin{array}{l}\text { Seeds of } P \text {. granatum are grounded } \\
\text { together along with sugar dissolved in } \\
\text { water can be used for hepatitis }\end{array}$ & $\begin{array}{l}\text { Ouelbani 2016, Ikram \& } \\
\text { Hussain } 1978\end{array}$ \\
\hline Ranunculaceae & Anemone hepatica L. & Leaves & $\begin{array}{l}\text { Triterpenoids, saponins, steroids, } \\
\text { lactones, fats and oils, saccharides, } \\
\text { and alkaloids }\end{array}$ & Infusion is used for protection of liver & $\begin{array}{l}\text { Hao \& Xio,2017, Bonet } \\
\text { et al. } 1999\end{array}$ \\
\hline
\end{tabular}




\begin{tabular}{|c|c|c|c|c|c|}
\hline & Nigella sativa L: & Seeds & $\begin{array}{l}\text { Thymoquinone (TQ), } \\
\text { thymohydroquinone (THQ), } \\
\text { dithymoquinone, thymol, carvacrol, } \alpha \\
\text { and } \beta \text {-pinene, d-limonene, } \mathrm{d} \text { - } \\
\text { citronellol, } p \text {-cymene volatile oil }\end{array}$ & Decoction is used for jaundice & $\begin{array}{l}\text { Kidane et al. 2018, } \\
\text { Forouzanfar et al. } 2014\end{array}$ \\
\hline \multirow[t]{2}{*}{ Rhamnaceae } & Ziziphus jujuba Mill. & Fruit & Sterols, flavonoids, cerebrosides & $\begin{array}{l}\text { Used for treatment of jaundice, hepatitis, } \\
\text { and other liver disorders }\end{array}$ & Kumar \& Choyal 2012 \\
\hline & Ziziphus mauritiana Lam. & Leaves & $\begin{array}{l}\text { Betulinic aldehyde, betulinic acid, } \\
\text { ceanothic acid, frangufoline, } \\
\text { spinosin and stearic acid }\end{array}$ & For cure of hepatic disorders & $\begin{array}{l}\text { Zaman et al. 2018, Guo } \\
\text { et al. } 2014\end{array}$ \\
\hline \multirow[t]{4}{*}{ Rosaceae } & Prunus domestica L. & Fruit & $\begin{array}{l}\text { D-galactose, D-mannose, L- } \\
\text { arabinose, D-xylose, glucoronic } \\
\text { acid, prudumestin }\end{array}$ & $\begin{array}{l}\text { Extract of dried fruit of P. domestica } \\
\text { soaked are used for hepatitis. }\end{array}$ & $\begin{array}{l}\text { Prajapati et al. } 2006, \\
\text { Pieroni et al. } 2014\end{array}$ \\
\hline & Rubus ellipticus Smith & Fruit & $\begin{array}{l}\text { Gallic acid, catechin, chlorogenic } \\
\text { acid and caffeic acid }\end{array}$ & $\begin{array}{l}\text { Charbohydrates, Alkaloids, Saponins, } \\
\text { Tannins, Flavonoids, Tritorpinoids }\end{array}$ & $\begin{array}{l}\text { Ahmad et al. 2017, } \\
\text { Badhni et al. 2015, } \\
\text { Sharma \& Kumar } 2011\end{array}$ \\
\hline & $\begin{array}{l}\text { Prunus jacquemontii } \\
\text { Hook. f. }\end{array}$ & Fruit & Proanthocyanidin, epicatechin & For the cure of hepatitis $C$ & $\begin{array}{l}\text { Hussain et al. 2018, } \\
\text { Pant et al. } 1992\end{array}$ \\
\hline & Prunus spinosa L. & Fruit & $\begin{array}{l}\text { Anthocyanin, hydroxycinnamic } \\
\text { acids }\end{array}$ & Hepatoprotector' & $\begin{array}{l}\text { Sabatani et al. } 2020 \text {, } \\
\text { Pieroni et al. } 2004\end{array}$ \\
\hline \multirow[t]{3}{*}{ Rubiaceae } & $\begin{array}{l}\text { Aegle marmdos (L.) } \\
\text { Correa }\end{array}$ & $\begin{array}{l}\text { Leaves, } \\
\text { roots }\end{array}$ & Tannin, flavonoids, and steriods & $\begin{array}{l}\text { Decoction of leaves and unripe fruit is } \\
\text { used for treatment of jaundice and other } \\
\text { liver disorder. }\end{array}$ & Kumar \& Choyal 2012 \\
\hline & Morinda citrifolia $L$. & Fruit & $\begin{array}{l}\text { Scopoletin, octanoic acid, } \\
\text { potassium, vitamin C, terpenoids, } \\
\text { alkaloids, } \\
\text { anthraquinones,nordamnacanthal, } \\
\text { morindone, rubiadin }\end{array}$ & For the cure of hepatitis & $\begin{array}{l}\text { Samani \& Kopaei, } \\
\text { 2018, Su et al. } 2005\end{array}$ \\
\hline & Rubia cordifolia L. & Roots & $\begin{array}{l}\text { Anthraquinone, lucidin } \\
\text { primeveroside, ruberythric acid and } \\
\text { three new anthraquinones, }\end{array}$ & Decoction is used for liver problems & $\begin{array}{l}\text { Ahmad et al. } 2017 \text {, } \\
\text { Itokawa } 1983\end{array}$ \\
\hline \multirow[t]{2}{*}{ Rutaceae } & $\begin{array}{l}\text { Citrus sinensis (L.) } \\
\text { Osbeck }\end{array}$ & Fruit & $\begin{array}{l}\text { Atripliside } B, \beta \text {-sitosterol, and } \beta \text { - } \\
\text { sitosterol-3-O- } \beta \text {-D-glucopyranoside }\end{array}$ & For the cure of hepatitis C & $\begin{array}{l}\text { Samani \& Kopaei, } \\
\text { 2018, Ceuterick et al. } \\
2008, \text { Saleem et al. } \\
2010\end{array}$ \\
\hline & Citrus limon (L.) Osbeck & Fruit & $\begin{array}{l}\beta \text {-Pinene, Limonene, Linalool and } \\
\alpha \text {-Terpineol }\end{array}$ & $\begin{array}{l}\text { Dink the juice with honey for liver } \\
\text { infections }\end{array}$ & $\begin{array}{l}\text { Hsouna et al. 2017, } \\
\text { Ellena et al. } 2012\end{array}$ \\
\hline
\end{tabular}




\begin{tabular}{|c|c|c|c|c|c|}
\hline Saxifragaceae & $\begin{array}{l}\text { Saxifraga melanocentra } \\
\text { Franch }\end{array}$ & Aerial parts & $\begin{array}{l}\text { Kaermpferol (1), } \\
\text { quercetin, kaempferol, beta } \\
\text { glucoside, quercetin, -D-glucoside }\end{array}$ & For the cure of hepatitis $C$ & $\begin{array}{l}\text { Samani \& Kopaei, } \\
\text { 2018, Zuo et al. } 2005\end{array}$ \\
\hline Scrophulariaceae & $\begin{array}{l}\text { Picrohiza kurooa Royle } \\
\text { ex.Benth }\end{array}$ & Rhizome & $\begin{array}{l}\text { Kutkin, a bitter glycoside which } \\
\text { contains two C-9 iridoid glycosides- } \\
\text { Picroside I and Kutakoside. }\end{array}$ & $\begin{array}{l}\text { Rhizome powder is used in the treatment } \\
\text { of liver troubles and jaundice }\end{array}$ & Kumar \& Choyal 2011 \\
\hline Solanaceae & Solanum nigrum L. & Leaves & $\begin{array}{l}\text { Gentisic acid, luteolin, apigenin, } \\
\text { kaempferol, m-coumaric acid. } \\
\text { anthocyanidin }\end{array}$ & To cure hepatitis, A,B,C & Hussain et al. 2016 \\
\hline Urticaceae & Arietaria judaica L. & Leaves & $\begin{array}{l}\text { Piperitone, camphor and ethyl } \\
\text { cinnamate }\end{array}$ & Used as antiviral & Maxia et al. 2008 \\
\hline \multirow[t]{2}{*}{ Zingiberaceae } & Curcuma longa L. & Root & $\begin{array}{l}\text { Ar-turmerone, } \beta \text {-sesquiphellandrene } \\
\text { and curcumenol, curcumin }\end{array}$ & Decoction is used for hepatitis C & $\begin{array}{l}\text { Abduel-Lateef et al. } \\
2016, \text { Sandhu et al. } \\
2005\end{array}$ \\
\hline & $\begin{array}{l}\text { Zingiber officinale } \\
\text { Roscoe }\end{array}$ & Root & $\begin{array}{l}\text { 6-gingerol, } 6 \text { shogaol, and 6-paradol, } \\
\text { zingiberene and bisabolene, } \\
\text { gingerols and shogaols }\end{array}$ & Decoction is used for jaundice & $\begin{array}{l}\text { Feng et al. 2011, } \\
\text { Heinrich et al. } 1998\end{array}$ \\
\hline
\end{tabular}




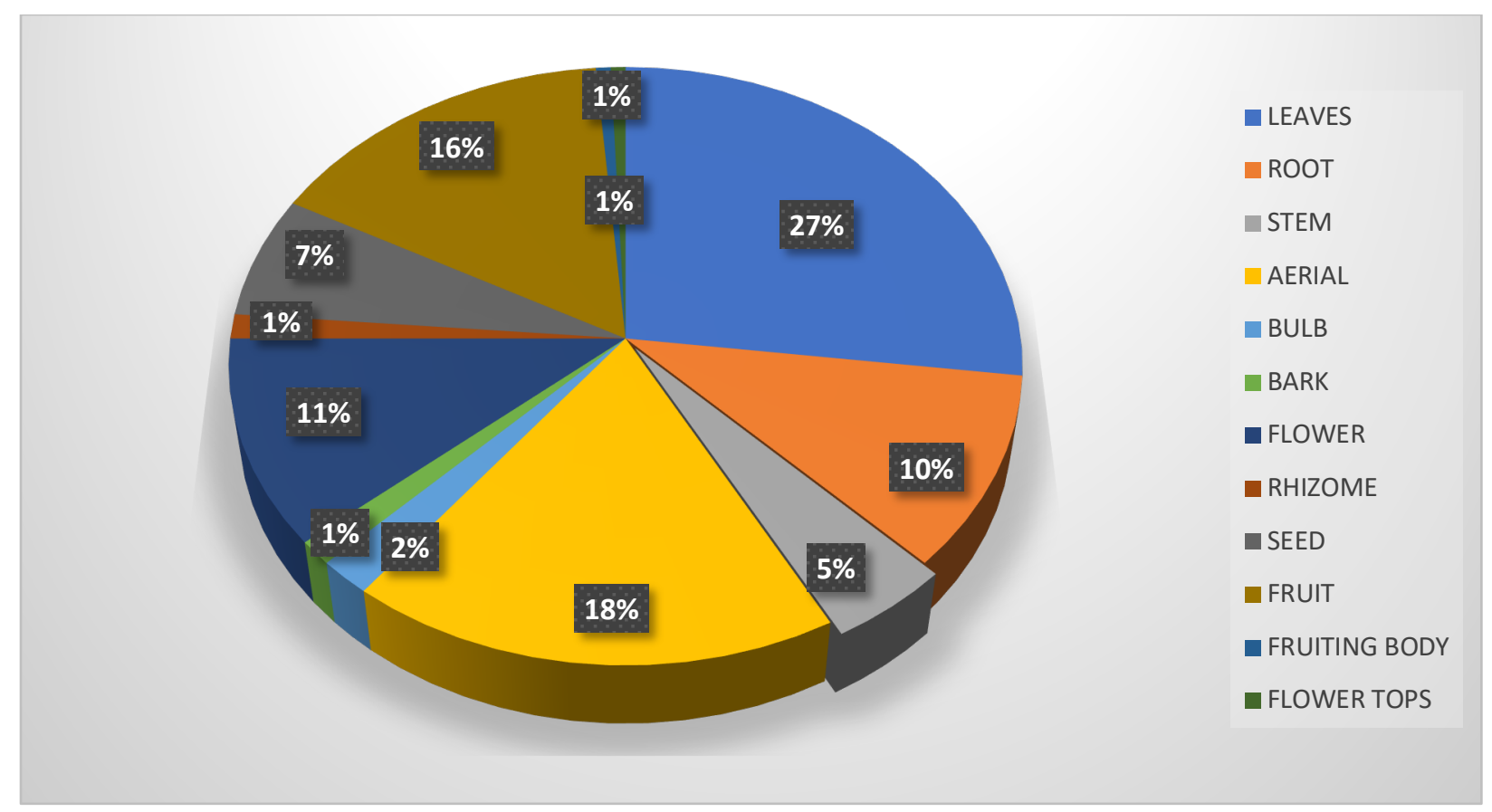

Figure 2. Medicinal Plants Part used for the treatment of hepatitis

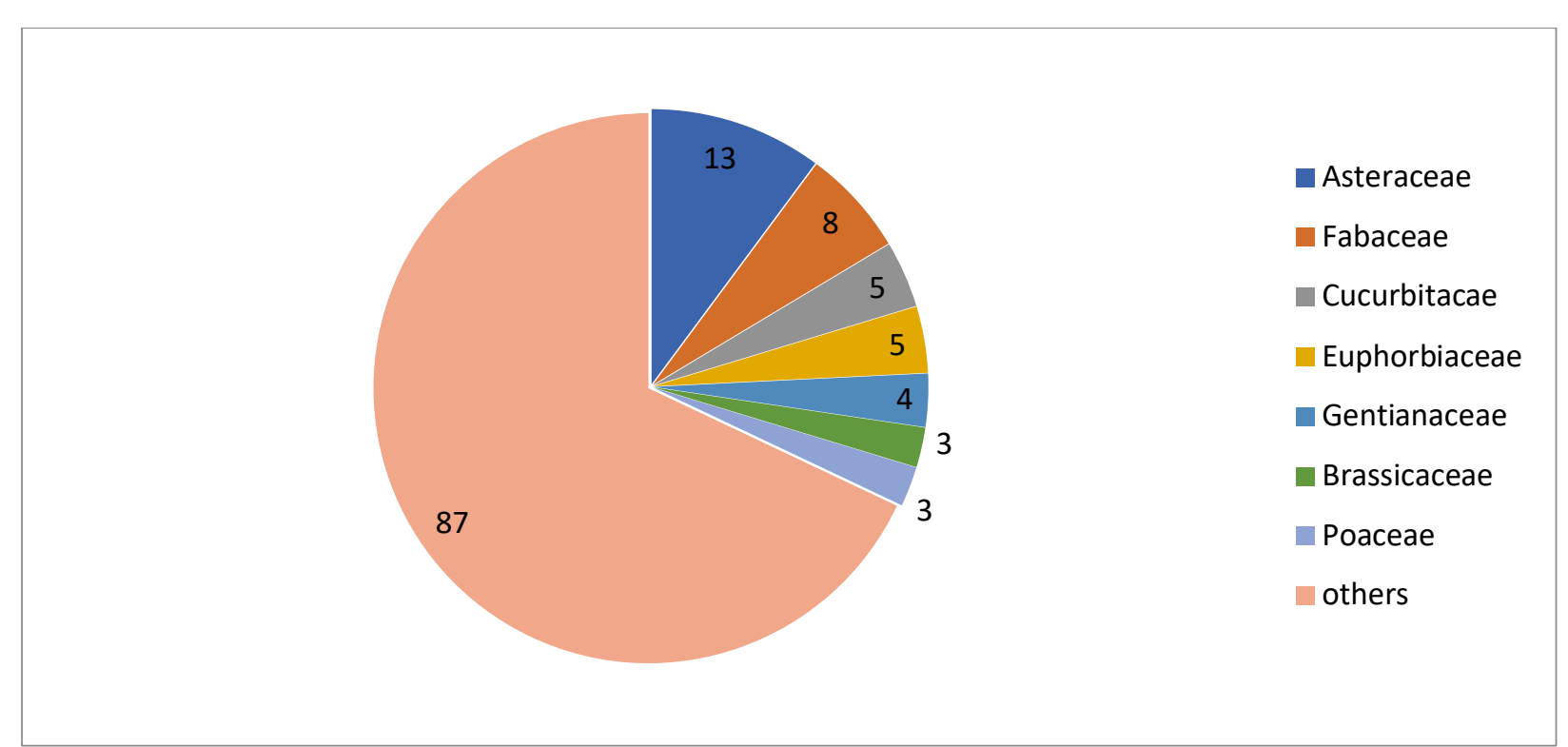

Figure 3. Frequency of the most represented botanical families (Number species)

In order to assess how geographical and ecological factors may have affected indigenous practices, the recorded data were subjected to regional comparison. The medicinal plants were divided into four groups (a) Pakistan (b) Asia (c) Europe (d) Rest of the world. Jaccard's similarity index was applied to determine similarities on the medicinal flora used for the cure of different types of hepatitis in different cultural communities all over the world. According to the Jaccard's index of similarity, the Pakistani communities used sum total of 58 species, Asia 56 species, Europe 24 species and rest of the world 18 species for the cure of hepatitis. Between Pakistani and Asia community's 14 species (12\%) were found to be the same while between Asia and Europe 5 species $(6.2 \%)$ were found in common. The similarity between Pakistan and Europe communities was 7 species $(11 \%)$. The current study documented 6 species $(7.8 \%)$ similarity between Pakistan and rest of the world and 5 species (6.2\%) were shared between Asia and Europe. The similarity between Asia and Rest of the world were recorded as 4 (5.4\%) Figure 4. The maximum similarities were observed between Pakistan and Asia (12 \%). 


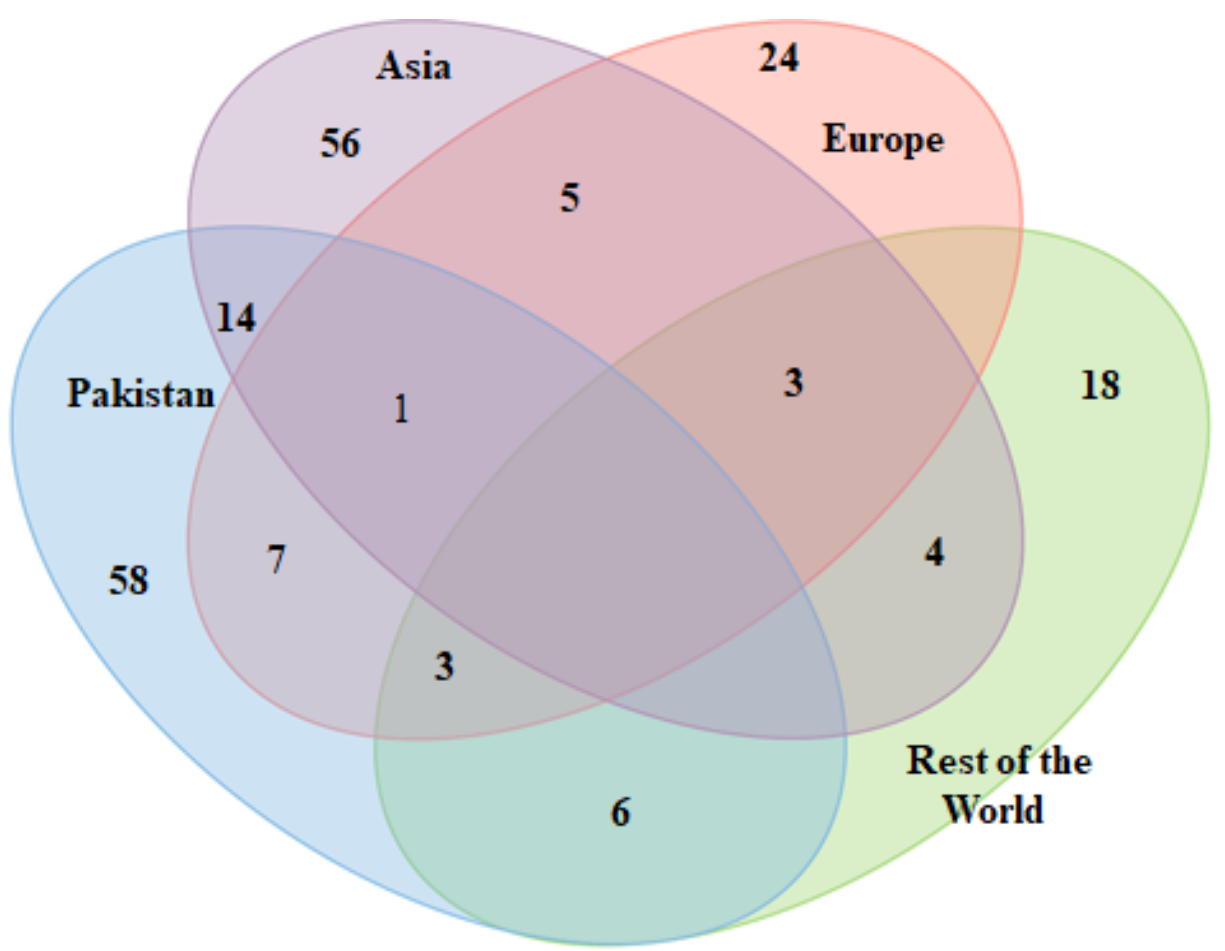

Figure 4. Venn diagram showing the comparison among the four studied regions (number of recorded plant species).

Silybum marianum (Milk thistle) has been used to treat liver diseases since the $16^{\text {th }}$ century. Its major constituents are the flavonoids silibinin, silidianin, silichristin, and isosilibinin of which silibinin is the biologically most active compound and used for standardization of pharmaceutical products (Wagner et al. 1976). The pharmacological profile of Silymarin has been well defined and hepatoprotective properties of Silymarin were investigated both in vitro and in vivo. Glycyrrhizin is an extract of the Glycyrrhiza glabra, used in medicine traditionally to relieve bronchitis, gastritis and jaundice. The compounds present in glycyrrhizin are glycyrrhetic acid, flavonoids, hydroxycoumarins, and betasitosterol. For treatment of chronic hepatitis, a standardized extract containing glycyrrhizin, cysteine and glycine is organized as Stronger NeoMinophagen C (Stickel \& Schuppan 2007). The plants of the genus Phyllanthus are found in most tropical and sub-tropical countries and have long been used to treat chronic liver diseases. Phyllantins, hypophyllantins and several polyphenoles are major constituents of which chemical and pharmacological properties are well described (Calixto et al. 1998). The active compounds within Phyllanthus showed anti-viral activities against hepatitis $B$ virus infection through mRNA transcription and replication (Venateswaran et al. 1987). Recently, a number of trials were evaluated regarding Phyllanthus extract preparations in a systematic review which led to clinical evidences for the treatment of chronic hepatitis B (Liu et al. 2001). To cure liver disorders numerous herbal mixtures are identified through search in traditional Oriental Medicine Database (Chen et al. 1998). Among these, extracts from Plantago asiatica appear to exert hepatoprotective activity without significant toxicity. In United States, for both acute and chronic hepatitis with focal necrosis and portal fibrosis Lycopodium serratum has been considerably used based on duration of intake. It has sleep-inducing properties (Woolf et al. 1994, Picciotto et al. 1998). In U.S. ephedrine in Ma Hung (Ephedra sp.) used to lose weight. A person with acute hepatitis used Ma-Hung for only 3 weeks, he developed anti-nuclear antibodies and muscle anti-bodies (Nadir et al. 1996).

Many plant species have been examined for a broadspectrum activity to cure liver disorders (AsadiSamani et al. 2015). Panax ginseng belongs to family Araliaceae. Roots of ginseng inhibit toxin hepatic damage by decreasing vital genes expression, which is essential for normal liver functions (Hafez et al. 2017). Cynara scolymus (artichoke) of the Asteraceae family, a species, is traditionally used for the treatment of digestive disorders, moderate hyperlipidemia and liver and biliary diseases. C. scolymus leaf extract has been used for its hepatoprotective effects (Gebhardt 1997). By studying literature it has been confirmed that $C$. scolymus was important in maintaining normal liver function parameters, maintaining liver redox status, as manifested by a significant increase in the activities of antioxidant enzymes and a 
reduction in glutathione accompanied by inhibition of lipid peroxidation (LPO) and protein oxidation, decreased nitric oxide and tumor necrosis factor alpha, and stabilized membrane in untested paracetamol-intoxicated rats (Ali et al. 2012). Hedyotis diffusa and some of its active constituents have a wide variety of reported pharmacological activities, including anticancer, chemo preventive, hepatoprotective, antiviral, antibacterial, antidiabetic, antioxidant, and gastroprotective properties (Qi et al. 2015). Nigella sativa belongs to family Ranunculaceae is used traditionally for the different disorders such as asthma, cough, bronchitis, headache, rheumatism, fever, influenza, and eczema. Antioxidant activity and resolution of hepato-renal toxicity have been reported for $\mathrm{N}$. sativa seeds (Al-Ghamadi 2001). Juice of Ananas comosus (L.) Merr (Bromeliaceae) commonly known as pineapple, has liver protective action (Mohamad et al. 2015) by controlling different protein expression, antioxidant levels and liver marker enzymes against paracetamol-induced toxicity. Fruit seeds of Cassia fistula (golden shower tree of family (Fabaceae) have protective potential against hepatotoxinsinduced liver damage and have non-significant effect on hematological parameters (lqbal et al. 2016). Fruiting bodies of Oudemansiella radicata Relhan ex $\mathrm{Fr}$ an edible mushroom and belong to the family Physalacriaseae has hepatoprotective activity by antioxidant mechanisms attributed to heteropolysaccharides (mannose, glucose and galactose) prepared from the mushroom (Liu et al. 2017). Heteropolysaccharides (arabinose and galactose) from Ziziphus jujube Mill commonly known as red date family Rhamnaceae has been involved in liver protective activity via alleviating liver marker enzymes (Liu et al. 2015). Phyllanthus amarus Schum et Thonn (Bhuia amla in Hindi) is a medicinal herb belonging to the family Euphorbiacae. The whole plant is used commonly in central and southern India. It is an ayurvedic herb and has a wide range of traditional uses including jaundice (Bhragwal et al. 2010). Monochoria hastata is used for the treatment of hepatitis in tehsil Shakargarh, Punjab, Pakistan (Ali et al. 2020). Ullah et al. (2010) showed that the root of Gynosporia royleana is used to cure of hepatitis $C$. The leaves of Pegonia cretica are used to treat hepatitis. The ethnobotanical survey in Taiwan showed that there are (69) different types of raw drugs and (107 species) of therapeutic taxa, which are highly applied for the cure of hepatitis by increasing the liver safety and to increase the ability of the liver to resist hepatitis (Lin \& Kan 1990). For the treatment of viral hepatitis people in Dembia district in Ethiopia used the medicinal plants viz., Adhatoda schimperiana, Phytolacca dodecandra and Calpurnia aurea for the treatment of hepatitis by taking their juice directly
(Tadele et al. 2015; Asmamaw and Achamyeleh 2018;). Similarly, the trace elements present in the leaves of plants and in other tissues of herbal taxa used in control of hepatitis as reported by some authors (Malede et al. 2020). Yao et al. (2020) reported that the one of the most famous tradition Chinese medicinal herb Abrus cantoniensis Hance (AC) is used to cure hepatitis B virus (HBV) by inhibiting the production of HBV DNA as well as Hepatitis Be Antigen (HBsAg), and Hepatitis B surface antigen (HBsAg). From India (Monika et al. 2020) also showed that some medicinal plants i.e. the leaf and flower part of the Pandanus fascicularis Lam, the root of the Berberis aristata DC and the whole plant of Oxalis corniculata $L$ is used for the treatment of hepatitis. Farzaei et al. (2020) showed that the Glycyrrhiza glabra (Licorice) used for the treatment of hepatitis, besides from the treatment this plant also famous for the treatment of cough and sore throat.

Many other medicinal plant species are traditionally used to treat hepatitis. Plants derived natural products such as flavonoids, terpenes, protein, lignans and alkaloids to inhibit the replication cycle of DNA and RNA viruses (Prajapati et al. 2006). Argyrolobium roseum (Fabaceae) produces vitex (flavonoid) and D-pinitol (monosaccharide) and these two compounds are reported to control hepatitis C (Gupta et al. 2005, Ram et al. 2007). Medicinal plants such as Adiantum capillus-veneris, Argyrolobium roseum, Equisetum debile, Carissa opaca, Phyllanthus emblica, Segeretia brandrethiana, Viola serpens, Plantago ovate, Woodfordia fruticosa, Saccharum officinarium and Prunus domestica have more recently been reported for the treatment of hepatitis. The medicinal plant Adiantum capillus-veneris (Adiantaceae) comprising chemical constituents, which are used for the treatment of hepatitis. It is important to confirm that which plant parts are used in liver diseases because in most of the phytochemical investigations, the secondary metabolites are different in plants parts. Different types of plants used by the local communities of Kalat and Huzdar region of Baluchistan, Pakistan traditionally to cure various types of hepatitis (Table 1). In addition, it has also been reported that, mostly plants used as a treatment of Hepatitis in the Soan Valley Salt Range Pakistan (Tareen et al. 2010, Bibi et al. 2014). The main natural products report from these plants are flavonoids, terpenoids, tannins, volatile oil, gallic acid (Khanum et al. 2013). The main secondary metabolites reported from Berberis lycium (Berberidaceae) have been shown active in curing hepatitis, as has Equisetum debile (Equisetaceae), Cucumis sativus (Cucurbitaceae), Cuscuta reflexa (Convolvulaceae) showed anti hepatitis activity 
((Abbasi et al. 2010, Khan et al. 2010). The seeds of Hordeum vulgare contain chemical constituents for the cure of hepatitis e.g. arginine, histidine, lysine, tyrosine, methionine (Abassi et al. 2009). Some compounds reported from Morus alba (Moraceae) fruit treat jaundice and hepatitis. Morus nigra (Moraceae) produce olcanolic acid, apigenin, cyclocommunol, morusin and cyclomorusin which are used to cure jaundice (Wang et al. 2007). Oxalis corniculata (Oxalidaceae) leaves contain, glyxylic acid, oxalic acid, vitexin, isovitexin, vitamin $C$ and tocopherols that are useful to treat hepatitis A and B types. The main constituents of the fruits of Phyllanthus emblica (Euphorbiaceae) anti-hepatitis compounds such as alanine, aspartic acid, glutamic acid, proline, fats, and iron (Abassi et al. 2009). The root extracts of $P$. niruri (Euphorbiaceae) are used to treat liver diseases; the extracts of this plant are reported to contain phyllanthine, flavonoids such as quercetin and isoquercetin. Prunus domestica (Rosaceae) fruit contains D-galactose, D-mannose, L-arabinose, D-xylose, glucoronic acid, flavonoid, and prudomestin. Punica granatum L. (Punicaceae) contain citric acid, sorbitol, mannitol, pelletierine, isoquercetrin, glucose, sucrose, maltose and oxalic acid in their fruits and seeds (Ikram 1978) and both plants show anti-hepatitis activity. Silybum marianum (Asteraceae) is most important for the treatment of hepatitis. Extracts of Silybum marianum (milk thistle) have been recognized as "Liver tonic" for centuries. Milk thistle has been reported to have protective effects on liver and to greatly improve its function (Hackett et al. 2013). Silybum marianum leaves are reported to contain flavoligrans, silybin, isosilybin and silymarin and these compounds have application to cure jaundice. The main compounds reported from Silybum marianum are the flavolagnans silybin, silydianin, and silychristine collectively called as silymarin. Silybin is the most important compound with range of biological activities. Moreover, Silymarin found in the whole plant but its more concentrated in fruits $6 \%$ in ripe fruit) and seeds. In addition, extracts of milk thistle have been recognized as liver tonic (Dhiman et al. 2005). Another plant, Ziziphus mauritiana (Rhamnaceae) contains twenty-one compounds, including ten triterpenoids, two sterols, six flavonoid and three cerebrosides, these were isolated from the fruit of Zizyphus mauritiana for the disorder of liver diseases. Melia azedarach is found to be useful to treat hepatitis. Many of these plants show antioxidant activity, possibly explaining their hepatoprotective activity (Moradi et al. 2016). The crude powder of Mimosa pudica prevents liver cell necrosis and lysosomal latency by normalizing serum biochemical parameters against carbon tetrachloride induced hepatotoxicity (Qadir et al. 2017). The roots of Boerhavia diffusa, commonly known as 'Punarnava', are used by a large number of tribes in India for the treatment of various hepatic disorders. The hepatoprotective activity of different parts of Boerhavia diffusa L. (Nyctaginaceae) such as root and aerial parts was evaluated against lbuprofen persuade hepatotoxicity in Wistar albino rats (Maity et al. 2015).

\section{Conclusions}

Our study showed that many medicinal plants have been used since ancient time to cure hepatitis worldwide. Our literature review will help the scientific community to identify anti-hepatitis plants in order to isolate novel anti-hepatitis compounds.

\section{Declarations}

List of abbreviations: HAV:Hepatitis A Virus, HBV:Hepatitis B Virus, HCV:Hepatitis C Virus, WHO:World Health Organization, LPO:lipid peroxidation, DNA:Deoxy-ribo Nucleic Acid, RNA:Ribo-Nucleic Acid, HIV:Human Immune Virus

Ethics statement: Thius study ia pure literature reveiw, and no ethics approval was required.

Conflict of Interest: The authors declare no conflicts of interest.

Funding: This research did not receive any specific grant from funding agencies in the public, commercial, or not-for-profit sectors.

Authors' contributions: Wahid Hussain designed the project wrote initial draft, Saima Batool, Sabeha Bahar, Bibi Shahida, Bushra Bangash helped in data collection. Amjad Ali, Hidayat Hussain, Iftikhar Ali, Maroof Ali and Rainer W. Bussmann helped in the revision of the manuscript. All the authors approved the final manuscript after revision.

\section{Literature cited}

Abbas W, Hussain W, Hussain W, Badshah L, Hussain K, Pieroni A. 2020. Traditional wild vegetables gathered by four religious groups in Kurram District, Khyber Pakhtunkhwa, North-West Pakistan. Genetic Resources and Crop Evolution I 116. 10.1007/s10722-020-00926-3.

Abbas Z, Khan SM, Abbasi AM, Pieroni A, Ullah Z, lqbal M, Ahmad Z. 2016. Ethnobotany of the Balti community, Tormik valley, Karakorum range, Baltistan, Pakistan. Journal of Ethnobiology and Ethnomedicine 12(1):38.

Abbasi AM, Khan MA, Ahmad M, Zafar M, Jahan S, Sultana S. 2010. Ethnopharmacological application of medicinal plants to cure skin diseases and in folk cosmetics among the tribal communities of NorthWest Frontier Province. Pakistan. Journal of Ethnopharmacology 128(2):322-335. 
Abbasi AM, Khan MA, Ahmad M, Zafar M, Khan H, Muhammad N, Sultana S. 2009. Medicinal plants used for the treatment of jaundice and hepatitis based on socio-economic documentation. African Journal of Biotechnology 8(8):1643-1650.

Abdel-Lateef E, Mahmoud F, Hammam O, ElAhwany E, El-Wakil E, Kandil S, Hassenein H. 2016. Bioactive chemical constituents of Curcuma longa L. rhizomes extract inhibit the growth of human hepatoma cell line (HepG2). Acta Pharmaceutica 66(3):387-398.

Addo EM, Chai HB, Hymete A, Yeshak MY, Slebodnick C, Kingston DG, Rakotondraibe LH. 2015. Antiproliferative constituents of the roots of Ethiopian Podocarpus falcatus and structure revision of 2a-hydroxynagilactone $F$ and nagilactone I. Journal of Natural Products 78(4):827-835.

Adebowale KO, Adedire CO. 2006. Chemical composition and insecticidal properties of the underutilized Jatropha curcas seed oil. African Journal of Biotechnology 5(10):901.

Adnan M, Jan S, Mussarat S, Tariq A, Begum S, Afroz A, Shinwari ZK. 2014. A review on ethnobotany, phytochemistry and pharmacology of plant genus Caralluma R. B r. Journal of Pharmacy and Pharmacology 66(10):1351-1368.

Agelet A, Valles J. 2003. Studies on pharmaceutical ethnobotany in the region of Pallars (Pyrenees, Catalonia, Iberian Peninsula). Part II. New or very rare uses of previously known medicinal plants. Journal of Ethnopharmacology 84(2-3):211-227.

Ahamed T, Mondal K, Khan M I, Munni TN, Alam T, Islam R. 2018. Ethnomedicinal survey of plants used by the folk medicinal practitioner (FMP) in the Jamalpur sadar Upazila, Jamalpur district, Bangladesh. Asian Journal of Medical and Biological Research 4(4):422-426.

Ahmad A, Ahmad B, Ali A, Ahmad Y. 2009. Seroprevalence of HBsAg and anti-HCV in general healthy population of Swat district with frequency of different HCV Genotypes. Pakistan Journal of Medical Sciences 25(5):744-748.

Ahmad K, Pieroni A (2016) Folk knowledge of wild food plants among the tribal communities of Thakhte-Sulaiman Hills, North-West Pakistan. Journal of Ethnobiology and Ethnomedicine 12:7. https:// doi.org/10.1186/s13002-016-0090-2.

Ahmad K, Weckerle CS, Nazir A (2019) Ethnobotanical investigation of wild vegetables used among local communities in northwest Pakistan. Acta Societatis Botanicorum Poloniae 88(1):3616. https://doi.org/10.5586/asbp.3616.
Ahmad KS, Hamid A, Nawaz F, Hameed M, Ahmad F, Deng J, Akhtar N, Wazarat A, Mahroof S. 2017. Ethnopharmacological studies of indigenous plants in Kel village, Neelum Valley, Azad Kashmir, Pakistan. Journal of Ethnobiology and. Ethnomedicine 13:68. doi.org/10.1186/s13002-0170196-1

Al Jahid A, Elamrani A, Lahlou FA, Hmimid F, Bourhim N, Blaghen M, Eddine JJ. 2017. Chemical Composition and Antibacterial Activity of the Essential Oil Isolated from the Seeds of Moroccan Artemisia campestris L. Journal of Essential Oil Bearing Plants 20(2):375-384.

Al-Ghamdi MS. 2001. The anti-inflammatory, analgesic and antipyretic activity of Nigella sativa. Journal of Ethnopharmacology 76(1):45-48.

Al-Snafi AE. 2015. The chemical constituents and pharmacological effects of Chenopodium album-An overview. International Journal of Pharmacological Screening Methods 5(1):10-17.

Al-Snafi AE. 2016. A review on chemical constituents and pharmacological activities of Coriandrum sativum. IOSR Journal of Pharmacy 6(7):17-42.

Al-Snafi AE. 2018. Pharmacological importance of Herniaria glabra and Herniaria hirsuta-A review. INDO American Journal of Pharmaceutical Research 5(4):2167-2175.

Alam I, Alam I, Ali I, Ali M. 2014. Weight loss in HCV patients can be used as a surrogate marker for evaluation of interferon (IFN- $\alpha$ ) treatment efficacy-A prospective pilot study. Pakistan Journal of Pharmaceutical Sciences 27:571-576.

Álamo JM, Olivares C, Jiménez G, Bernal C, Marín LM, Tinoco J, Gómez MÁ. 2013. December). Donor characteristics that are associated with survival in liver transplant recipients older than 70 years with grafts. In Transplantation proceedings 45(10):36333636).

Ali A, Badshah L, Hussain F. 2018a Ethnobotanical Appraisal and Conservation Status of Medicinal Plants in Hindukush Range, District Swat, Pakistan. Journal of Herbs, Spices \& Medicinal Plants. doi:10.1080/10496475.2018.1510456.

Ali K. Khan N, Rahman IU, Khan W, Ali M, Uddin N, Nisar M. 2018b. The ethnobotanical domain of the Swat Valley, Pakistan. J. Ehnobiol. Ethnomed 14(1):39.

Ali M, Iqbal IM, Shabbir A, Khan ZUD, Khan MTA. 2020. Ethnomedicinal studies on aquatic plants of tehsil Shakargarh, Punjab, Pakistan. Journal of Medicinal Plants 8(1):15-19. 
Ali ZY, Atia HA, Ibrahim NH. 2012. Possible hepatoprotective potential of Cynara scolymus, Cupressus sempervirens and Eugenia jambolana against paracetamol-induced liver injury:in-vitro and in-vivo evidence. Natural Sciences 10(1):75-86.

Alshawsh MA, Abdulla MA, Ismail S, Amin ZA. 2011. Hepatoprotective effects of Orthosiphon stamineus extract on thioacetamide-induced liver cirrhosis in rats. Evidence-based complementary and alternative medicine.

Ammar NM, El-Kashoury ESA, Abou El-Kassem LT, Abd El-Hakeem RE. 2013. Evaluation of the phenolic content and antioxidant potential of Althaea rosea cultivated in Egypt. Journal of The Arab Society for Medical Research 8(2):691-694.

Andrade JM, Faustino C, Garcia C, Ladeiras D, Reis CP, Rijo P 2018. Rosmarinus officinalis L.:an update review of its phytochemistry and biological activity. Future science OA 4(4):FSO283.

Appendino G, Belliardo F, Nano GM, Stefenelli S. 1982. Sesquiterpene lactones from Artemisia genipi Weber:isolation and determination in plant material and in liqueurs. Journal of Agricultural and Food Chemistry 30(3):518-521.

Asadi-Samani M, Kafash-Farkhad N, Azimi N, Fasihi A, Alinia-Ahandani E, Rafieian-Kopaei M. 2015. Medicinal plants with hepatoprotective activity in Iranian folk medicine. Asian Pacific Journal of Tropical Biomedicine 5(2):146-157.

Ashfaq UA, Idrees S. 2014. Medicinal plants against hepatitis C virus. World Journal of Gastroenterology:WJG 20(11):2941.

Bader GN, Mir PA, Naqash A, Ali T, Wadoo R, Ali S. 2017. Phytochemical screening and evaluation of Hepatoprotective potential of Swertia petiolata against thioacetamide induced hepatotoxicity in rats. International Journal Current Research 9(06):5273747.

Badhani A, Rawat S, Bhatt ID, Rawal RS. 2015. Variation in Chemical Constituents and Antioxidant Activity in Yellow Himalayan (Rubus ellipticus Smith) and Hill Raspberry (Rubus niveus T hunb). Journal of food biochemistry 39(6):663-672.

Bai N, He KAN, Roller M, Zheng B, Chen X, Shao Z, Zheng Q. 2008. Active compounds from Lagerstroemia speciosa, insulin-like glucose uptakestimulatory/inhibitory and adipocyte differentiationinhibitory activities in 3T3-L1 cells. Journal of Agricultural and Food Chemistry 56(24):1166811674.

Balick MJ, Elisabetsky E, Laird SA. (Eds.). 1996. Medicinal resources of the tropical forest:biodiversity and its importance to human health. Columbia University Press.

Bandaranayake WM. 2006. Quality control, screening, toxicity, and regulation of herbal drugs. Modern Phytomedicine:25-57.

Barros L, Pereira E, Calhelha RC, Dueñas M, Carvalho AM, Santos-Buelga C, Ferreira IC. 2013. Bioactivity and chemical characterization in hydrophilic and lipophilic compounds of Chenopodium ambrosioides L. Journal of Functional Foods 5(4):1732-1740.

Batiha GES, Alkazmi LM, Wasef LG, Beshbishy AM, Nadwa EH, Rashwan EK. 2020. Syzygium aromaticum L.( Myrtaceae):Traditional uses, bioactive chemical constituents, pharmacological and toxicological activities. Biomolecules 10(2):202.

Bhatia H, Sharma YP, Manhas R, Kumar K. 2014. Ethnomedicinal plants used by the villagers of district Udhampur, J\&K, India. Journal of Ethnopharmacology 151:1005-1018.

Bhragual DD, Kumar N, Garg VK, Sharma PK. 2010. Review on plants havin $g$ hepatoprotective activity. Journal of Pharmacy Research 3(9):2077-2082.

Bibi S, Sultana J, Sultana H, Malik, RN. 2014a. Ethnobotanical uses of medicinal plants in the highlands of Soan Valley, Salt Range, Pakistan. Journal of Ethnopharmacology 155(1):352-361.

Bibi T, Ahmad M, Tareen RB, Tareen NM, Jabeen R, Rehman SU, Sultana S, Zafar M, Yaseen G. 2014b Ethnobotany of medicinal plants in district Mastung of Balochistan province-Pakistan. Journal of Ethnopharmacology 157:79-89. doi:10.1016/j.jep.2014.08.042

Boh B, Berovic M, Zhang J, Zhi-Bin L. 2007. Ganoderma lucidum and its pharmaceutically active compounds. Biotechnology Annual Review 13:265301.

Calixto JB, Santos AR, Filho VC, Yunes RA. 1998. A review of the plants of the genus Phyllanthus:their chemistry, pharmacology, and therapeutic potential. Medicinal Research Reviews 18(4):225-258.

Ceuterick M, Vandebroek I, Pieroni A. 2011. Resilience of Andean urban ethnobotanies:a comparison of medicinal plant use among Bolivian and Peruvian migrants in the United Kingdom and in their countries of origin. Journal of Ethnopharmacology 136(1):27-54.

Ceuterick M, Vandebroek I, Torry B, Pieroni A. 2008. Cross-cultural adaptation in urban ethnobotany:the Colombian folk pharmacopoeia in London. Journal of Ethnopharmacology 120(3):342-359. 
Chen L, Chen R, Wei K. 1992. Constituents of tannins from Euphorbia prostrata Ait. Zhongguo Zhong yao za zhi= Zhongguo zhongyao zazhi= China Journal of Chinese Materia Medica 17(4):2256.

Chen SL, Morgan TR. 2006. The natural history of hepatitis $\mathrm{C}$ virus $(\mathrm{HCV})$ infection. International Journal of Medical Sciences 3(2):47-52.

Chen TSN, Chen PS. Liver in traditional Chinese medicine. Journal of Gastroenterology and Hepatology 1998:13:437-42

Choo QL, Kuo G, Weiner AJ, Overby LR, Bradley DW, Houghton M. 1989. Isolation of a cDNA clone derived from a blood-borne non-A, non-B viral hepatitis genome. Science 244(4902):359-362.

Corrêa GM, Alcântara AFDC. 2012. Chemical constituents and biological activities of species of Justicia: a review. Revista Brasileira de Farmacognosia, 22(1):220-238.

Cusheri A. 2002. Acute and chronic viral hepatitis. In:Cusheri A, Steel JC, Moosa AR, editors. Essential surgical practice. 5th ed. London:Oxford University Press 2:334-5.

Da Cheng H, Xiao-Jie G, Pei GX. 2015. Phytochemical and biological research of Papaver pharmaceutical resources. Medicinal Plants:Chemistry Biology and Omics 217-251.

De Melo MLS, Narain N, Bora PS. 2000. Characterisation of some nutritional constituents of melon (Cucumis melo hybrid AF-522) seeds. Food Chemistry 68(4):411-414.

Dhiman RK, Chawla YK. 2005. Herbal medicines for liver diseases. Digestive Diseases and Sciences 50(10):1807-1812.

EASL International consensusconference on hepatitis B.13-14 September, 2002 Geneva , Switzerland. Journal of Hepatology 2003;38:533-40

Egea T, Signorini MA, Bruschi P, Rivera D, Obón C, Alcaraz F, Palazón JA. 2015. Spirits and liqueurs in European traditional medicine:Their history and ethnobotany in Tuscany and Bologna (Italy). Journal of Ethnopharmacology 175:241-255.

Ellena R, Quave CL, Pieroni A. 2012. Comparative medical ethnobotany of the Senegalese community living in Turin (Northwestern Italy) and in Adeane (Southern Senegal). Evidence-Based Complementary and Alternative Medicine, 2012.

Ercisli S, Orhan E. 2007. Chemical composition of white (Morus alba), red (Morus rubra) and black (Morus nigra) mulberry fruits. Food Chemistry 103(4):1380-1384.
Escudero NL, De Arellano ML, Fernández S, Albarracín G, Mucciarelli S. 2003. Taraxacum officinale as a food source. Plant Foods for Human Nutrition 58(3):1-10.

Farooq A, Amjad MS, Ahmad K, Altaf M, Umair M, Abbasi MA. 2019. Ethnomedicinal knowledge of the rural communities of Dhirkot, Azad Jammu and Kashmir, Pakistan. Journal of Ethnobiology and Ethnomedicine 15 (1):45. doi:10.1186/s13002-0190323-2.

Farzaei MH, Bayrami Z, Farzaei F, Aneva I, Das SK, Patra JK, Abdollahi M. 2020. Poisoning by Medical Plants. Archives of Iranian Medicine 23(2):117.

Fawad K. 2016 response to anti -viral therapy in district Mardan, Khyber Pakhtunkhawa, Pakistan. Asian Pacific Journal of Cancer Prevention 1037-40.

Feng T, Su J, Ding ZH, Zheng YT, Li Y, Leng Y, Liu JK. 2011. Chemical constituents and their bioactivities of "Tongling White Ginger"(Zingiber officinale). Journal of agricultural and food chemistry 59(21):11690-11695.

Forouzanfar F, Bazzaz BSF, Hosseinzadeh H. 2014. Black cumin (Nigella sativa) and its constituent (thymoquinone):a review on antimicrobial effects. Iranian journal of basic medical sciences 17(12):929.

Foster S. 1991. Milk thistle Silybum marianum (No. Folleto 14309).

Gandomi H, Abbaszadeh S, JebelliJavan A, Sharifzadeh A. 2014. Chemical Constituents, Antimicrobial and Antioxidative Effects of Trachyspermum ammi Essential Oil. Journal of Food Processing and Preservation 38(4):1690-1695.

Gebhardt R. 1997. Antioxidative and protective properties of extracts from leaves of the artichoke (Cynara scolymus L.) against hydroperoxideinduced oxidative stress in cultured rat hepatocytes. Toxicology and Applied Pharmacology 144(2):279286.

Gewali MB, Awale S. 2008. Aspects of traditional medicine in Nepal. Japan:Institute of Natural Medicine. University of Toyama.

Giday M, Asfaw Z, Woldu Z, Teklehaymanot T. 2009. Medicinal plant knowledge of the Bench ethnic group of Ethiopia:an ethnobotanical investigation. Journal of Ethnobiology and Ethnomedicine 5(1):34.

Gokavi SS, Malleshi NG, Guo M. 2004. Chemical composition of garden cress (Lepidium sativum) seeds and its fractions and use of bran as a functional ingredient. Plant Foods for Human Nutrition 59(3):105-111. 
Govind P, Pandey SP. 2011. Phytochemical and toxicity study of Emblica officinalis (Amla). International Research Journal of Pharmacy 2(3):270-272.

Gunes S, Savran A, Paksoy MY, Kosar M, Cakılcıoglu U. 2017. Ethnopharmacological survey of medicinal plants in Karaisalı and its surrounding (Adana-Turkey). Journal of Herbal Medicine 8:68-75. doi:10.1016/j.hermed.2017.04.002.

Guo S, Duan JA, Zhao JL, Qian DW, Zhang WJ. 2014. Chemical constituents from seeds of Ziziphus mauritiana. Zhong yao cai= Zhongyaocai $=$ Journal of Chinese Medicinal Materials 37(3):432-435.

Gupta OP, Ahmed Z, Bhagat A, Gupta KK, Handa SS. 2005. U.S. Patent No. 6,869,625. Washington, DC:U.S. Patent and Trademark Office.

Gupta PC, Sharma N, Rao CV. 2012. A review on ethnobotany, phytochemistry and pharmacology of Fumaria indica (Fumitory). Asian Pacific Journal of Tropical Biomedicine 2(8):665-669.

Gupta VK, Sharma SK. 2006. Antioxidants neutralise the effect of free radicals through different ways and may.Appears in Collections:NPR Vol.5(4),1346-51.

Gurudeeban S, Satyavani K, Ramanathan T. 2010. Bitter apple (Citrullus colocynthis):An overview of chemical composition and biomedical potentials. Asian Journal of Plant Sciences 9(7):394.

Habib-ur-Rehman, Yasin KA, Choudhary MA, Khaliq N, Atta-Ur-Rahman, Choudhary MI, Malik S. 2007. Studies on the chemical constituents of Phyllanthus emblica. Natural Product Research 21(9):775-781.

Hackett ES, Twedt DC, Gustafson DL. 2013. Milk thistle and its derivative compounds:a review of opportunities for treatment of liver disease. Journal of Veterinary Internal Medicine 27(1):10-16.

Hadjiakhoondi A, Vatandoost $H$, Khanavi $M$, Sadeghipour Roodsari HR, Vosoughi M, Kazemi M, Abai MR. 2006. Fatty acid composition and toxicity of Melia azedarach L. fruits against malaria vector Anopheles stephensi. Iranian Journal of Pharmaceutical Sciences 2(2):97-102.

Hadžifejzović N, Kukić-Marković J, Petrović S, Soković M, Glamočlija J, Stojković D, Nahrstedt A. 2013. Bioactivity of the extracts and compounds of Ruscus aculeatus L. and Ruscus hypoglossum L. Industrial crops and products 49:407-411.

Hafez MM, Hamed SS, El-Khadragy MF, Hassan ZK, Al Rejaie SS, Sayed-Ahmed MM., Al-Shabanah OA. 2017. Effect of ginseng extract on the TGF- $\beta 1$ signaling pathway in $\mathrm{CCl}$ 4-induced liver fibrosis in rats. BMC Complementary and Alternative Medicine 17(1):1-11.
Hakim ST, Kazmi SU, Bagasra O. 2008. Seroprevalence of hepatitis B and C genotypes among young apparently healthy females of KarachiPakistan. Libyan Journal of Medicine 3(2):66-70.

Hao DC, Gu X, Xiao P. 2017. Anemone medicinal plants:ethnopharmacology, phytochemistry and biology. Acta Pharmaceutica Sinica B 7(2):146-158.

Haq F, Ahmad H, Alam M. 2011. Traditional uses of medicinal plants of Nandiar Khuwarr catchment (district Battagram), Pakistan. Journal of Medicinal Plants Research 5(1):39-48.

Haq I, Hussain M. 1993. Medicinal plants of Mansehra. Hamdard Medicus 36(3):63-100.

He K, Cao TW, Wang HL, Geng CA, Zhang XM, Chen JJ. 2015. Chemical constituents of Swertia patens. Zhongguo Zhong yao za zhi= Zhongguo zhongyao zazhi= China journal of Chinese Materia Medica 40(20):4012-4017.

Heinrich M, Ankli A, Frei B, Weimann C, Sticher O. 1998. Medicinal plants in Mexico:Healers' consensus and cultural importance. Social Science \& Medicine 47(11):1859-1871. doi:10.1016/S02779536(98)00181-6.

Hsouna AB, Halima NB, Smaoui S, Hamdi N. 2017. Citrus limon essential oil:chemical composition, antioxidant and antimicrobial activities with its preservative effect against Listeria monocytogenes inoculated in minced beef meat. Lipids in Health and Disease 16(1):146.

Hussain I, Shah M, Khan MS, Saqlian M. 2013. Prevalence of hepatitis $C$ in selected patients in Parachinar, Kurrum Agency, Pakistan. International Journal of Biosciences 3(3):142-54.

Hussain J, Munir M, Hassan Z, Bano N, Arshad S, Ahmad VU. 2010. Tanacetamide D:a new ceramide from Tanacetum artemisioides. Helvetica Chimica Acta 93(2):350-353.

Hussain W, Badshah L, Ullah M. 2018. Quantitative study of medicinal plants used by the communities residing in Koh-e-Safaid Range, northern PakistaniAfghan borders. Journal of Ethnobiology and Ethnomedicine 14(1):30 . doi.org/10.1186/s13002018-0229-4

Hussain W, Hussain J, Ali R, Khan I, Shinwari ZK, Nascimento IA. 2012. Tradable and conservation status of medicinal plants of KurramValley. Parachinar, Pakistan. Journal of Applied Pharmaceutical Science 2(10):66.

Idm'hand E, Msanda F, Cherifi K. 2020. Ethnobotanical study and biodiversity of medicinal plants used in the Tarfaya Province, Morocco. Acta 
Ecologica Sinica 40 doi:10.1016/j.chnaes.2020.01.002.

(2):134-144.

Ikram M, Hussain SF. 1978. Compendium of medicinal plants. P.C.S.I.R Laboratories, Peshawer 23-14

Ikram M, Nowshad F. 1977. Constituents of Quercus infectoria. Planta medica 31(03):286-287.

Iqbal I, Afzal M, Aftab MN, Manzoor F, Kaleem A, Kaleem A. 2016. Hepatotoxicity of Cassia fistula extracts in experimental chicks and assessment of clinical parameters. Kuwait Journal of Science 43(3).

Ishtiaq S, Ahmad M, Hanif U, Akbar S, Kamran SH. 2014. Phytochemical and in vitro antioxidant evaluation of different fractions of Amaranthus graecizans subsp. silvestris (Vill.) Brenan. Asian Pacific Journal of Tropical Medicine 7:S342-S347.

Jaccard P. 1902. Lois de distribution florale dans la zone alpine. Bulletin of the the Vaudois Society of Natural Sciences 38:69-130

Jaime MFV, Redko F, Muschietti LV, Campos RH, Martino VS, Cavallaro LV. 2013. In vitro antiviral activity of plant extracts from Asteraceae medicinal plants. Virology Journal 10(1):245.

Jan HA, Wali S, Ahmad L, Jan S, Ahmad N, Ullah N. 2017. Ethnomedicinal survey of medicinal plants of Chinglai valley, Buner district, Pakistan. European Journal of Internal Medicine 13:64-74.

Kadir MF, Sayeed MSB, Mia M. 2012. Ethnopharmacological survey of medicinal plants used by indigenous and tribal people in Rangamati, Bangladesh. Journal of Ethnopharmacology 144(3):627-637. doi:10.1016/j.jep.2012.10.003.

Kassaye KD, Amberbir A, Getachew B, Mussema Y. 2006. A historical overview of traditional medicine practices and policy in Ethiopia. Ethiopian Journal of Health Development 20(2):127-134.

Kato N. 2000. Genome of human hepatitis C virus (HCV), gene organization, sequence diversity, and variation. Microbial and Comparative Genomics 5:129-51.

Kayani S, Ahmad M, Sultana S, Shinwari ZK, Zafar M, Yaseen G, Bibi T. 2015. Ethnobotany of medicinal plants among the communities of Alpine and Subalpine regions of Pakistan. Journal of Ethnopharmacology 164:186-202. https://doi.org/ 10.1016/j.jep.2015.02.004

Kebela D, Achamyeleh H. 2018. Assessment of medicinal plants and their conservation status in case of Daligaw Kebela, Gozamen Werda, East Gojjam Zone. J Biodivers Biopros Dev 5:170-175.
Khan AA, Ahmad H, Ghafoor US, Begum K, Khan Al. 2010. Frequency distribution of hepatitis in three districts of Hazara Division. Asian Journal 45:16-19.

Khan I, Najeebullah S, Ali M, Shinwari ZK. 2016. Phytopharmacological and ethnomedicinal uses of the Genus Berberis (Berberidaceae):A review. Tropical Journal of Pharmaceutical Research 15(9):2047-2057.

Khan S, Mirza KJ, Abdin MZ. 2010. Development of RAPD markers for authentication of medicinal plant Cuscuta reflexa. Eurasian Journal of Biosciences 1(4):1-7.

Khan ST, Riaz N, Afza N, Nelofar A, Malik A, Ahmed E, Hussain S. 2006. Studies on the chemical constituents of Prosopis cineraria. Journal of the Chemical Society of Pakistan 28(6):619-622.

Khanum R, Jahangir M, Abbasi MA, Mazhar F, Kausar S, Riaz T, Ajaib M. 2013. Phytochemical Screening and Antioxidant Evaluations of Different Fractions of Argyrolobium roseum. Asian Journal of Chemistry 25(13):14219-31.

Kidane L, Gebremedhin G, Beyene T. 2018. Ethnobotanical study of medicinal plants in Ganta Afeshum District, Eastern Zone of Tigray, Northern Ethiopia. Journal of Ethnobiology and Ethnomedicine 14:64. doi:10.1186/s13002-0180266-z.

Kitazato K, Wang Y, Kobayashi N. 2007. Viral infectious disease and natural products with antiviral activity. Drug Discoveries \& Therapeutics 1(1):14-22.

Konowalchuk J, Speirs JI. 1976. Virus inactivation by grapes and wines. Applied and Environmental Microbiology 32(6):757-763.

Konowalchuk J, Speirs JI. 1978. Antiviral effect of apple beverages. Applied and Environmental Microbiology 36(6):798-801.

Konowalchuk J, Speirs JI. 1978. Antiviral effect of commercial juices and beverages. Applied and Environmental Microbiology 35(6):1219-1220.

Kpodar MS, Lawson-Evi P, Bakoma B, EkluGadegbeku P, Agbonon A, Aklikokou A, Gbeassor M. 2015. Ethnopharmacological survey of plants used $\mathrm{n}$ the treatment of diabetes mellitus in south of Togo (Maritime Region). Journal of Herbal Medicine 5(3):147-152. doi:10.1016/j.hermed.2015.06.002.

Kumar CH, Ramesh A, Kumar JS, Ishaq BM. 2011. A review on hepatoprotective activity of medicinal plants. International journal of Pharmaceutical sciences and research 2(3):501.

Kumar KA, Sharvanee S, Patel J, Choudhary RK. 2010. Chemical composition and antimicrobial 
activity of the essential oil of Desmostachya bipinnata L. International Journal of Phytomedicine 2(4).

Kumar N, Choyal R. 2012. Traditional use of some plants of Hamirpur district of Himachal Pradesh for the treatment of jaundice, hepatitis and other liver disorders. International Journal of Theoretical \& Applied Sciences 4:201-205.

Kumar N, Kumar R, Kishore K. 2013. Onosma L.:A review of phytochemistry and ethnopharmacology. Pharmacognosy Reviews 7(14):140.

Kuo PC, Yang ML, Wu PL, Shih HN, Thang TD, Dung NX, Wu TS. 2008. Chemical constituents from Abutilon indicum. Journal of Asian natural products research 10(7):689-693.

Li D, Zuo H, Gao H, Wu L. 2004. Study on the chemical constituents of Sophora flavescens Ait. Journal of Shenyang Pharmaceutical University 21(5):346-348.

Liang HX, Dai HQ, Fu HA, Dong XP, Adebayo AH, Zhang LX, Cheng YX. 2010. Bioactive compounds from Rumex plants. Phytochemistry Letters 3(4):181-184.

Lin CC, Kan WS. 1990. Medicinal plants used for the treatment of hepatitis in Taiwan. The American journal of Chinese medicine 18(01):35-43.

Liu G, Liu X, Zhang Y, Zhang F, Wei T, Yang M, Zhao Z. 2015. Hepatoprotective effects of polysaccharides extracted from Zizyphus jujuba cv. Huanghetanzao. International Journal of Biological Macromolecules 76:169-175.

Liu J, Lin H, Mclntosh H. 2001. Genus Phyllanthus for chronic hepatitis B virus infection:a systematic review. J Viral Hep 8:368-466.

Liu Q, Zhu M, Geng X, Wang H, Ng TB. 2017. Characterization of polysaccharides with antioxidant and hepatoprotective activities from the edible mushroom Oudemansiella radicata. Molecules 22(2):234.

Lok AS, Mc Mohan BJ. 2009. Chronic hepatitis B. Hepatology 50(03):661-662.

Mahmood A, Malik RN, Shinwari ZK. 2013. Indigenous knowledge of medicinal plants from Gujranwala district, Pakistan. Journal of Ethnopharmacology 148:714-723. doi:10.1016/j.jep.2013.05.035.

Maity T, Maity S, Pahari N, Kar DR, Ganguli S. 2015. A review on hepatic diseases and development of herbal drugs for the treatment of liver complications. World Journal of Pharmaceutical Research 4:677691.
Malede M, Tefera M, Mehari B. 2020. Trace Metals in the Leaves of Selected Plants Used to Treat Hepatitis in Dembia, Ethiopia. Journal of Herbs, Spices \& Medicinal Plants 26(1):101-112.

Malla B, Gauchan DP, Chhetri RB. 2015. An ethnobotanical study of medicinal plants used by ethnic people in Parbat district of western Nepal. Journal of Ethnopharmacology 165:103-117.

Mangrio SM, Dahot MU, Leghari SM. 1995. Chemical constituents of Equisetum debile Roxb. Pakistan Journal of Plants Sciences 1(1):41-48.

Marrelli M, Amodeo V, Statti G, Conforti F. 2019. Biological properties and bioactive components of Allium cepa L. Focus on potential benefits in the treatment of obesity and related comorbidities. Molecules 24(1):119.

Maxia A, Lancioni MC, Balia AN, Alborghetti R, Pieroni A, Loi MC. 2008. Medical ethnobotany of the Tabarkins, a Northern Italian (Ligurian) minority in south-western Sardinia. Genetic Resources and Crop Evolution 55(6):911-924.

Mazutti M, Mossi AJ, Cansian RL, Corazza ML, Dariva C, Oliveira JV. 2008. Chemical profile and antimicrobial activity of Boldo (Peumus boldus Molina) extracts obtained by compressed carbon dioxide extraction. Brazilian Journal of Chemical Engineering 25(2):427-434.

Medeiros PMD, Soldati GT, Alencar NL, Vandebroek I, Pieroni A, Hanazaki N, de Albuquerque U. 2012. The use of medicinal plants by migrant people:adaptation, maintenance, and replacement. Evidence-Based Complementary and Alternative Medicine, 2012.

Mkaddem M, Bouajila J, Ennajar M, Lebrihi A, Mathieu F, Romdhane M. 2009. Chemical composition and antimicrobial and antioxidant activities of Mentha longifolia L. and $M$. viridis essential oils. Journal of Food Science 74(7):M358M363.

Monika DM, Bisht PS, Chaturvedi P. 2020. Medicinal Uses of Traditionally Used Plants in Bhatwari Block, District Uttarkashi, Uttarakhand, India. Journal of Scientific Research 64(1).

Moradi MT, Asadi-Samani M, Bahmani M, Shahrani M. (2016. Medicinal plants used for liver disorders based on the Ethnobotanical documents of Iran:A Review. International Journal of PharmTech Research 9(5):407-415.

Moussaieff A, Shein NAA, Tsenter J, Grigoriadis S, Simeonidou C, Alexandrovich AG, Munoz E. 2008. Incensole acetate:a novel neuroprotective agent 
isolated from Boswellia carterii. Journal of Cerebral Blood Flow \& Metabolism 28(7):1341-1352.

Moyer LA, Mast EE, Alter MJ. 1999. Hepatitis C, routine serologic testing and diagnosis. American Family Physician 59:79-88.

Muley BP, Khadabadi SS, Banarase NB. 2009. Phytochemical constituents and pharmacological activities of Calendula officinalis L. (Asteraceae):a review. Tropical Journal of Pharmaceutical Research:8(5).

Mustafa B, Hajdari A, Krasniqi F, Hoxha E, Ademi H, Quave CL, Pieroni A. 2012. Medical ethnobotany of the Albanian Alps in Kosovo. Journal of Ethnobiology and Ethnomedicine 8(1):6.

Nadir A, Agrawal S, King PD, Marshall JB. 1996. Acute hepatitis associated with the use of a Chinese herbal product, Ma-Huang. American Journal of Gastroenterology 91:1436-8.

Nahrstedt A, Butterweck V. 1997. Biologically active and other chemical constituents of the herb of Hypericum perforatum L. Pharmacopsychiatry 30(S 2):129-134.

Nasri N, Tlili N, Elfalleh W, Cherif E, Ferchichi A, Khaldi A, Triki S. 2011. Chemical compounds from Phoenician juniper berries (Juniperus phoenicea). Natural Product Research 25(18):1733-1742.

Negi JS, Singh P, Joshi GP, Rawat MS, Bisht VK. 2010. Chemical constituents of Asparagus. Pharmacognosy Reviews 4(8):215.

Negi JS, Singh P, Rawat B. 2011. Chemical constituents and biological importance of Swertia:a review. Current Research in Chemistry 3(1):1-15.

Okunade, AL. 2002. Ageratum conyzoides L.(Asteraceae). Fitoterapia 73(1):1-16.

Omole RA, Gathirwa J, Akala H, Malebo H M, Machocho AK, Hassanali A, Ndiege IO. 2014. Bisbenzylisoquinoline and hasubanane alkaloids from Stephania abyssinica Dillon \& A. Rich. (Menispermeceae). Phytochemistry 103:123-128.

Ong HG, Kim YD. 2014. Quantitative ethnobotanical study of the medicinal plants used by the Ati Negrito indigenous group in Guimaras Island, Philippines. Journal of Ethnopharmacology 242:157:228. doi:10.1016/j.jep.2014.09.015.

Ouelbani R, Bensari S, Mouas TN, Khelifi D. 2016. Ethnobotanical investigations on plants used in folk medicine in the regions of Constantine and Mila (North-East of Algeria). J Ethnopharmacol194:196218. doi:10.1016/j.jep.2016.08.0.1
Ovanović O, Radulović., Stojanović G, Palić R, Zlatković ., Gudžić B. 2009. Chemical composition of the essential oil of Centaurium erythraea Rafn (Gentianaceae) from Serbia. Journal of Essential Oil Research 21(4):317-322.

Paltinean R, Toiu A, Wauters JN, Frederich M, Tits M, Angenot L, Crisan G. 2016. Phytochemical analysis of Fumaria officinalis L.(Fumariaceae). Farmacia 64(3):409-413.

Pant G, Nautiyal AK, Rawat MS, Sutherland JK, Morris GA. 1992. Identification and ab initio carbon13 NMR assignment of a proanthocyanidin from Prunus jacquemontii. Magnetic resonance in chemistry 30(13):S142-S147.

Paterska M, Bandurska H, Wysłouch J. 2017. Chemical composition of horse-chestnut (Aesculus) leaves and their susceptibility to chestnut leaf miner Cameraria ohridella Deschka \& Dimić. Acta Physiologiae Plantarum 39:105. https://doi.org/10.1007/s11738-017-2404-y

Pattiram PD, Lasekan O, Tan CP, Zaidul ISM. 2011. Identification of the aroma-active constituents of the essential oils of Water Dropwort (Oenanthe javanica) and'Kacip Fatimah'(Labisia pumila). International Food Research Journal 18(3).

Pereira AP, Ferreira IC, Marcelino F, Valentão P, Andrade PB, Seabra R, Pereira JA. 2007. Phenolic compounds and antimicrobial activity of olive (Olea europaea (L. Cv. Cobrançosa) leaves. Molecules 12(5):1153-1162.

Picciotto A, Campo N, Brizzolara R, Giusto R, Guido G, Sinelli N. 1998. Chronic hepatitis induced by Jin Bu Huan. Journal of Hepatology 28:165-7.

Pieroni A, Gray C. 2008. Herbal and food folk medicines of the Russlanddeutschen living in Künzelsau/Taläcker, South-Western Germany. Phytotherapy Research 22(7):889-901.

Pieroni A, Muenz $H$, Akbulut $M$, Başer KHC, Durmuşkahya C. 2005. Traditional phytotherapy and trans-cultural pharmacy among Turkish migrants living in Cologne, Germany. Journal of Ethnopharmacology 102(1):69-88.

Pieroni A, Muenz $H$, Akbulut $M$, Başer KHC, Durmuşkahya C. 2005. Traditional phytotherapy and trans-cultural pharmacy among Turkish migrants living in Cologne, Germany. Journal of Ethnopharmacology 102(1):69-88.

Pieroni A, Privitera S. 2014. Ethnobotany and its links to medical sciences and public health:quo vadis? Journal of phytotherapy 35(02):58-62.

Pieroni A, Quave CL, Giusti ME, Papp N. 2012. "We are Italians!":the hybrid ethnobotany of a Venetian 
diaspora in Eastern Romania. Human Ecology 40(3):435-451.

Pieroni A, Quave CL, Santoro RF. 2004. Folk pharmaceutical knowledge in the territory of the Dolomiti Lucane, inland southern Italy. Journal of Ethnopharmacology 95(2-3):373-384.

Pieroni A. 2008. Local plant resources in the ethnobotany of Theth, a village in the Northern Albanian Alps. Genetic Resources and Crop Evolution 55(8):1197-1214.

PMRC. Prevalence of hepatitis B and C in Pakistan, Islamabad. Pakistan Medical and Research Council 2008.

Prajapati ND, Purohit SS, Sharma AK, Kumar T. 2006. A Hand book of Medicinal Plants. 3rd Edition.

Qadir MI, Ahmad Z. 2017. Advances in hepatoprotective medicinal plants research. Bangladesh Journal of Pharmacology 12(3):229242.

Qi F, Zhao L, Zhou A, Zhang B, Li A, Wang Z, Han J. 2015. The advantages of using traditional Chinese medicine as an adjunctive therapy in the whole course of cancer treatment instead of only terminal stage of cancer. Bioscience Trends 9(1):16-34.

Ragasa CY, Guardamano JD, Tan MCS, Shen CC. 2016. Chemical Constituents of Brassica rapa Chinensis L. and Brassica rapa var. parachinensis (Baily) Hanelt. International Journal of Current Pharmaceutical Review and Research 7(5):316-318

Ram G, Bhan MK, Ahuja A, Meena SR, Kaul MK, Gupta KK, Jolly RL,Khajuria RK. 2007. Variability and selection on differentArgyrolobium roseum accessions for morphological traits and yield. Genetic Resources and Crop Evolution 54(03):649654.

Rao MR, Palada MC, Becker BN. 2004. Medicinal and aromatic plants in agro forestry systems. New vistas in agroforestry Springer Dordrecht 31:107122.

Rashid N, Gbedomon RC, Ahmad M, Salako VK, Zafar M, Malik K. 2018. Traditional knowledge on herbal drinks among indigenous communities in Azad Jammu and Kashmir, Pakistan. Journal of Ehnobiology and Ethnomedicine 14(1):16 doi:10.1186/s13002-018-0217-821

Riaz N, Nawaz SA, Mukhtar N, Malik A, Afza N, Ali S, Choudhary MI. 2007. Isolation and enzymeinhibition studies of the chemical constituents from Ajuga bracteosa. Chemistry \& bBodiversity 4(1):7283.
Rigat M, Bonet MÀ, Garcia S, Garnatje T, Valles J. 2007. Studies on pharmaceutical ethnobotany in the high river Ter valley (Pyrenees, Catalonia, Iberian Peninsula). Journal of Ethnopharmacology 113(2):267-277.

Saleem M, Farooq A, Ahmad S, Shafiq N, Riaz N, Jabbar A, Malik A. 2010. Chemical constituents of Citrus sinensis var. shukri from Pakistan. Journal of Asian natural products research 12(8):702-706. doi:10.1080/10286020.2010.489041.

Samani ZN, Kopaei MR. 2018. Effective Medicinal Plants in treating Hepatitis B. International Journal of Pharmaceutical Sciences and Research:9(9).

Sandhu DS, Heinrich M. 2005. The Use Of Health Foods, spices and other botanicals in the Sikh community in London. Phytotherapy Research:An International Journal Devoted to Pharmacological and Toxicological Evaluation of Natural Product Derivatives 19(7):633-642.

Seeger C, Mason WS, Lai MM. 2020. Molecular biology of hepatitis viruses. The Liver :Biology and Pathobiology 793-820.

Shabbir A, Shahzad M, Arfat Y, Ali L, Aziz RS, Murtaza G, Waqar SA. 2012. Berberis lycium Royle:A review of its traditional uses, phytochemistry and pharmacology Pakistan. African Journal of Pharmacy and Pharmacology 6(31):2346-2353.

Shah SA, Shah NA, Ullah S, Alam MM, Badshah H, Ullah S, Mumtaz AS. 2016. Documenting the indigenous knowledge on medicinal flora from communities residing near Swat River (Suvastu) and in high mountainous areas in Swat-Pakistan. Journal of Ethnopharmacolology 182:67-79.

Shang MY, Cai SQ, Lin WH, Wang MC, Park JH. 2002. Studies on chemical constituents from the seed of Trigonella foenum-graecum. Zhongguo Zhong yao za zhi= Zhongguo zhongyao zazhi= China Journal of Chinese Materia Medica 27(4):277279.

Sharma S, Kumar S. 2013. Phyllanthus reticulatus Poir an important medicinal plant:a review of its phytochemistry, traditional uses and pharmacological properties. International Journal of Pharmaceutical Sciences and Research 4(7):25282534.

Sharma US, Kumar A. 2011. In vitro antioxidant activity of Rubus ellipticus fruits. Journal of Advanced Pharmaceutical Technology \& Research 2(1):47.

Shivhare MK, Singour PK, Chaurasiya PK, Pawar RS. 2012. Trianthema portulacastrum L. (bishkhapra). Pharmacognosy reviews 6(12):132. 
Singh O, Khanam Z, Misra N, Srivastava MK. 2011. Chamomile (Matricaria chamomilla L.):an overview. Pharmacognosy Reviews 5(9):82.

Singh P, Khandelwal P, Sharma KK, Sharma MC. 2010. Cetyl triacontanoate and other constituents from Acacia jacquemontii and Kigelia pinnata. Journal of the Indian Chemical Society 87(11):1403.

Srikanth M, Swetha T, Veeresh B. 2012. Phytochemistry and pharmacology of Oxalis corniculata Linn.:A review. International Journal of Pharmaceutical Sciences and Research 3(11):4077.

Srivastav S, Singh P, Mishra G, Jha KK, Khosa RL. 2011. Achyranthes aspera-An important medicinal plant:A review. Journal of Natural Product and Plant Resources 1(1):1-14.

Stickel F, Schuppan, D. 2007. Herbal medicine in the treatment of liver diseases. Digestive and Liver Disease 39(4):293-304.

Su BN, Pawlus AD, Jung HA, Keller WJ, McLaughlin JL, Kinghorn AD. 2005. Chemical Constituents of the Fruits of Morinda citrifolia (Noni) and Their Antioxidant Activity. Journal of Natural Products 68(4):592-595.

Tadele A, Taddese M, Jeevanandham S. 2015. Determination of Some Toxic Heavy Metal Accumulation in Medicinal Plants Commonly Used in Gondar Area District, Northwestern Ethiopia. International Journal of Pharmacy and Analytical Research 4:399-405.

Tareen RB, Bibi T, Khan MA, Ahmad M, Zafar M, Hina S. 2010. Indigenous knowledge of folk medicine by the women of Kalat and Khuzdar regions of Balochistan, Pakistan. Pakistan Journal of Botany 42(3):1465-1485.

Thomas D, Leoutsakas D, Zabransky T and Kumar M. 2011. Hepatitis C in HIV infected individuals:cure and control, right now. Journal of the International AIDS Society 14(1):22.

Tilak JC, Adhikari S, Devasagayam TP. 2004. Antioxidant properties of Plumbago zeylanica, an Indian medicinal plant and its active ingredient, plumbagin. Redox Report 9:219-227 [PMID:15479566

DOI:10.1179/135100004225005976]

Touihri I, Kallech-Ziri O, Boulila A, Fatnassi S, Marrakchi N, Luis J, Hanchi B. 2019. Ecballium elaterium (L.) A. Rich. seed oil:Chemical composition and antiproliferative effect on human colonic adenocarcinoma and fibrosarcoma cancer cell lines. Arabian Journal of Chemistry 12(8):2347-2355.

Tuasha N, Petros B, Asfaw Z. 2018. Medicinal plants used by traditional healers to treat malignancies and other human ailments in Dalle District, Sidama Zone, Ethiopia. J. Ethnobiol. Ethnomed 14(1):15.

Tyler VE, Bradly R, Robers JE. 1981. Pharmacognosy, 8thedition. K.M. Varghese Company, Bombay, p. 250.

Uddin G, Rauf A, Rehman TU, Qaisar M. 2011. Phytochemical screening of Pistacia chinensis var. integerrima. Middle East Journal of Scientific Research 7(5):707-711.

Ullah K, GM, Shah J, Alam M, Hussain. 2020. Ethnobotany of the Medicinal Plants Used by Indigenous Communities in the Mountain of Shishikoh Valley, Hindukush Chitral, KP, Pakistan. Ukrainian Journal of Ecology., 10 (2), 92-105, doi:10.15421/2020_70

Ullah M, Zaynab M, Fatima M, Abbas S, Sharif Y, Farooq TH, Ullah I. 2018. Plants as Antidiabetic Agents:Traditional Knowledge to Pharmacological Validation. PSM Biological Research 3(3):111-119.

Ullah R, Hussain Z, Iqbal Z, Hussain J, Khan FU, Khan N, Hussain I. 2010. Traditional uses of medicinal plants in Darra Adam Khel NWFP Pakistan. Journal of Medicinal Plants Research 4(17):1815-1821.

Umair M, Altaf M, Abbasi AM. 2017. An ethnobotanical survey of indigenous medicinal plants in Hafizabad district, Punjab-Pakistan. PLOS ONE 12(6):e0177912. doi:10.1371/journal.pone.0177912.

Umar M, Khaar HB, Anwar F, Zahid M. 2002. Themanagement of acute viral bleeding by octreotide. Journal of Rawalpindi Medical College 4:14-16.

Van Damme P, Kokoska L. 2015. In Vitro Antistaphylococcal Effects of Embelia schimperi Extracts and Their Component Embelin with Oxacillin and Tetracycline.

Venateswaran PS, Millman I, Blumberg BS. 1987. Effects of an extract from Phyllanthus niruri on hepatitis B virus and woodchuck hepatitis viruses:in vitro and in vivo studies. Proceedings of the National Acadademy of Sciience USA 84:274-278.

Vitalini S, Iriti M, Puricelli C, Ciuchi D, Segale A, Fico G. 2013. Traditional knowledge on medicinal and food plants used in Val San Giacomo (Sondrio, Italy)—An alpine ethnobotanical study. Journal of Ethnopharmacology 145(2):517-529. doi:10.1016/j.jep.2012.11.024.

Wagner H, Seligmann O, Seitz M, Abraham D, Sonnenbichler J. 1976. Silydianin und Silychristin, zwei isomere Silymarine aus Silybum marianum L. Gaertn. (Mariendistel)/Silydianin and Silychristin, 
two Isomeric Silymarins from Silybum marianum L. Gaertn.( milk thistle). Zeitschrift fuer Naturforschung B 31(6):876-884.

Wang L, Wang HQ, Chen RY. 2007. Studies on chemical constituentsfrom bark of Morus nigra. Zhongguo Zhong Yao Za Zhi 32 (23):2497-2499.

Wangchuk P, Keller PA, Pyne SG, Taweechotipatr M, Tonsomboon A, Rattanajak R, Kamchonwongpaisan S. 2011. Evaluation of an ethnopharmacologically selected Bhutanese medicinal plants for their major classes of phytochemicals and biological activities. Journal of Ethnopharmacol 137:730-742.

Wangteeraprasert R, Lipipun V, Gunaratnam M, Neidle S, Gibbons S, Likhitwitayawuid K. 2012. Bioactive compounds from Carissa spinarum. Phytotherapy Research 26(10):1496-1499.

Wiedenfeld $\mathrm{H}$, Zych M, Buchwald W, Furmanow M. 2007. New compounds from Rhodiola kirilowii. Scientia Pharmaceutica 75(1):29-34.

Wkly Epidemiology Rec 2002;77:41.P. P. Sharma, A. M. Mujundar. Indian Journal of Traditional Knowledge. 2003;2:292-296.

World Health Organization. Hepatitis B.Geneva:WHO; 2000.

Yao X, Li Z, Gong X, Fu X, Xiao X, He M, Xu Z. 2020. Total saponins extracted from Abrus cantoniensis Hance suppress hepatitis $B$ virus replication in vitro and in rAAV8-1.3 HBV transfected mice. Journal of Ethnopharmacology 249:1123.

Yeşil Y, İnal İ. 2019. Traditional knowledge of wild edible plants in Hasankey (Batman Province, Turkey). Acta Societatis Botanicorum Poloniae 88(3):3633. doi:10.5586/asbp.3633.

Yöney A, Prieto JM, Lardos A, Heinrich M. 2010. Ethnopharmacy of Turkish-speaking Cypriots in greater London. Phytotherapy Research 24:731740.

Zahid M, Ali A, Ishurd O, Ahmed A, Ali Z, Ahmad VU, Pan Y. 2002. New cycloartane and flavonol glycosides from Corchorus depressus. Helvetica Chimica Acta 85(2):689-697.

Zaman SU, Ali K, Khan W, Ali M, Jan T, Nisar M. 2018. Ethno-botanical and geo-referenced profiling of medicinal plants of Nawagai Valley, District Buner (Pakistan). Biosystems Diversity 26(1) doi:10.15421/011809.

Zhou YX, Xin HL, Rahman K, Wang SJ, Peng C, Zhang H. 2015. Portulaca oleracea L.:a review of phytochemistry and pharmacological effects. BioMed Research International 2015.
Zuo G, Zhang Z, Chen L, Xu X. 2005. Chemical constituents of tibetan herbal medicine Saxifraga melanocentra. Acta Botanica Yunnanica 27(6):691694. 